\title{
Stress development, relaxation, and memory in colloidal dispersions: Transient nonlinear microrheology
}

R. N. Zia and J. F. Brady

Citation: J. Rheol. 57, 457 (2013); doi: 10.1122/1.4775349

View online: http://dx.doi.org/10.1122/1.4775349

View Table of Contents: http://www.journalofrheology.org/resource/1/JORHD2/v57/i2

Published by the The Society of Rheology

\section{Related Articles}

The lengths of thread-like micelles inferred from rheology

J. Rheol. 56, 1363 (2012)

Performance of mesoscale modeling methods for predicting rheological properties of charged polystyrene/water suspensions

J. Rheol. 56, 353 (2012)

Three-dimensional flow of colloidal glasses

J. Rheol. 56, 259 (2012)

Emergence of turbid region in startup flow of $\mathrm{CTAB} / \mathrm{NaSal}$ aqueous solutions between parallel plates

J. Rheol. 56, 245 (2012)

Nonlinear rheology and yielding in dense suspensions of hard anisotropic colloids

J. Rheol. 55, 1069 (2011)

\section{Additional information on J. Rheol.}

Journal Homepage: http://www.journalofrheology.org/

Journal Information: http://www.journalofrheology.org/about

Top downloads: http://www.journalofrheology.org/most_downloaded

Information for Authors: http://www.journalofrheology.org/author_information

\section{ADVERTISEMENT}

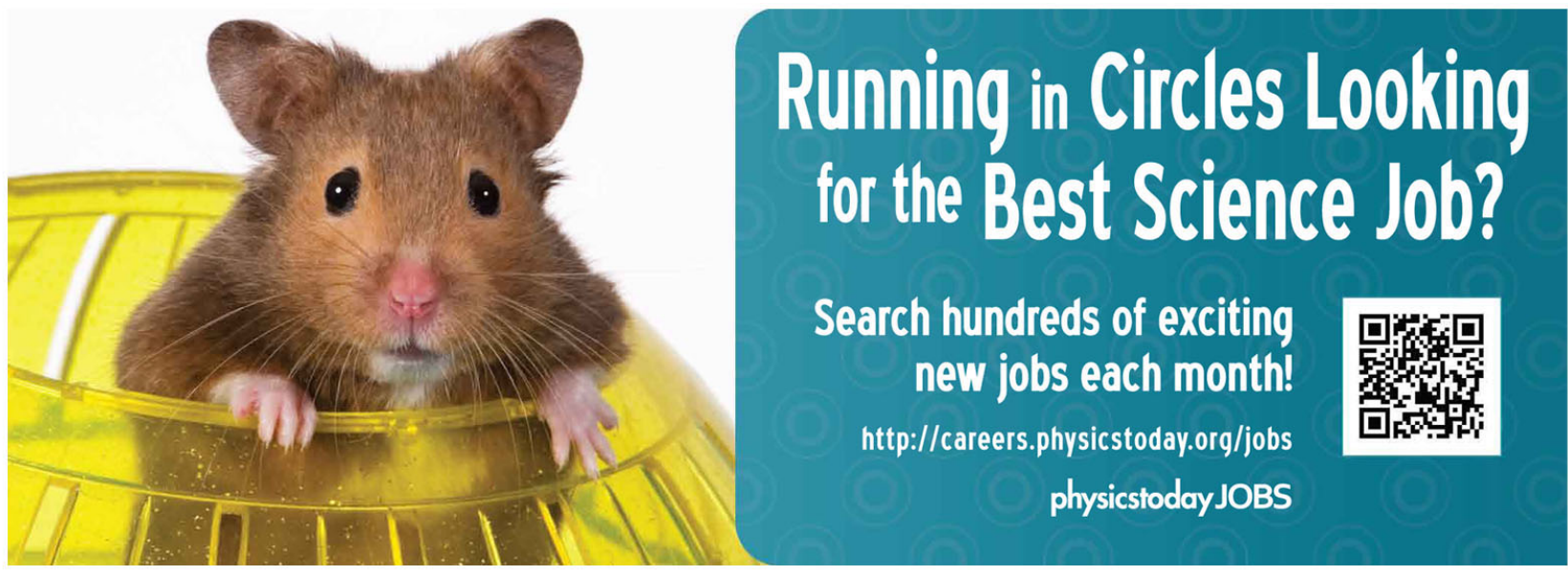




\title{
Stress development, relaxation, and memory in colloidal dispersions: Transient nonlinear microrheology
}

\author{
R. N. Zia ${ }^{\text {a) }}$ \\ Department of Mechanical Engineering, California Institute of Technology, \\ 1200 E California Boulevard, Pasadena, California 91125 \\ J. F. Brady \\ Departments of Mechanical Engineering and Chemical Engineering, \\ California Institute of Technology, 1200 E California Boulevard, \\ Pasadena, California 91125
}

(Received 17 May 2012; final revision received 27 November 2012; published 17 January 2013)

\begin{abstract}
Synopsis
The motion of a single Brownian particle in a complex fluid can reveal material behavior both at and away from equilibrium. In active microrheology, a probe particle is driven by an external force through a complex medium and its motion studied in order to infer properties of the embedding material. Most work in microrheology has focused on steady behavior and established the relationship between the motion of the probe, the microstructure, and the effective microviscosity of the medium. Transient behavior in the near-equilibrium, linear-response regime has also been studied via its connection to low-amplitude oscillatory probe forcing and the complex modulus; at very weak forcing, the microstructural response that drives viscosity is indistinguishable from equilibrium fluctuations. But important information about the basic physical aspects of structural development and relaxation in a medium is captured by startup and cessation of the imposed deformation in the nonlinear regime, where the structure is driven far from equilibrium. Here, we study theoretically and by dynamic simulation the transient behavior of a colloidal dispersion undergoing nonlinear microrheological forcing. The strength with which the probe is forced, $F^{\text {ext }}$, compared to thermal forces, $k T / b$, governs the dynamics and defines a Péclet number, $P e=F^{e x t} /(k T / b)$, where $k T$ is the thermal energy and $b$ is the colloidal bath particle size. For large $P e$, a boundary layer (in which unsteady advection balances diffusion) forms at particle contact on the time scale of the flow, $a / U$, where $a$ is the probe size and $U$ its speed, whereas the wake forms over $O(P e)$ diffusive time steps. Similarly, relaxation following cessation occurs over several time scales corresponding to distinct physical processes. For very short times, the time scale for relaxation is set by a boundary layer of thickness $\delta \sim(a+b) / P e$, and so $\tau \sim \delta^{2} / D_{r}$, where $D_{r}$ is the relative diffusivity between the probe of size $a$ and a bath particle. Nearly all stress relaxation occurs during this time. At longer times, the Brownian diffusion of the bath particles acts to close the wake on a time scale set by how long it takes a bath particle to diffuse laterally across it, $\tau \sim(a+b)^{2} / D_{r}$. Although the majority of the microstructural relaxation occurs during this wakehealing process, it does so with little change in the stress. Also during relaxation, the probe travels backward in the suspension; this recovered strain is proportional to the free energy stored in the
\end{abstract}

\footnotetext{
${ }^{\text {a)} A u t h o r ~ t o ~ w h o m ~ c o r r e s p o n d e n c e ~ s h o u l d ~ b e ~ a d d r e s s e d ; ~ e l e c t r o n i c ~ m a i l: ~ r z i a @ p r i n c e t o n . e d u ~}$
} 
compressed particle configuration, an indicator that the stress is proportional to the free energy density stored entropically in the microstructure. Theoretical results are compared with Brownian dynamics simulation where it is found that the dilute theory captures the correct behavior even for concentrated suspensions. Two modes of forcing are studied: Constant force and constant velocity. Results are compared to analogous macrorheology results for suspensions undergoing simple shear flow. (C) 2013 The Society of Rheology. [http://dx.doi.org/10.1122/1.4775349]

\section{INTRODUCTION}

Complex fluids are ubiquitous in natural and engineered systems, ranging from the intracellular compartment to pharmaceutical products to foodstuffs. Due to their multiphase structure, such materials exhibit a rich spectrum of flow-rate dependent responses that include shear thinning, shear thickening, and time-dependent behavior such as viscoelasticity and memory effects. Such nonequilibrium rheological behavior may be studied by inducing bulk material displacements via a shearing or extensional flow, for example. The imposed flow may be constant, in order to interrogate steady behavior, or it may vary in time-e.g., steady oscillation or sudden flow startup — in order to study viscoelastic or transient response. In traditional rheology, these perturbations are imposed over macroscopic length scales and therefore may exclude important materials of interest such as those in which steady or transient behaviors vary over the microscale or materials not available in sufficient quantities for bulk interrogation, e.g., rare biological fluids. An alternative approach is to interrogate the material over microscopic length scales-a microscale version of rheometry. This type of microscale probing, known as "microrheology," has a long history, tracing back to the work of Einstein (1906) and Perrin (1909); but advances in microscopy and other techniques in the last two decades [see, e.g., Crocker and Grier (1996)] has prompted considerable new study in the literature in recent years, in both experimental and theoretical investigations [MacKintosh and Schmidt (1999); Habdas et al. (2004); Meyer et al. (2006); Squires and Brady (2006); Khair and Brady (2006); Wilson et al. (2009); Zia and Brady (2010, 2012)].

In microrheology, the motion of a colloidal particle (or set of particles) is tracked in order to determine the properties of the surrounding medium [MacKintosh and Schmidt (1999)]. In passive microrheology, random displacements of tracers arise due to thermal fluctuations. These displacements are tracked and connected to the material modulus through a generalized Stokes-Einstein-Sutherland relation, a process which reveals the equilibrium and linear-response properties of the embedding material. Passive microrheology can be used to interrogate linear viscoelasticity [Mason and Weitz (1995)]; recent studies also account for the effect of probe size [Lu and Solomon (2002)] and shape [Khair and Brady (2008)]. Microrheological techniques have been used to study cells [Bausch et al. (1998); Guilford et al. (1995); Lau et al. (2003)], actin networks [Gisler and Weitz (1999); Ziemann et al. (1994)], gelatin [Freundlich and Seifriz (1923)], DNA and polyethylene oxide solutions [Mason et al. (2005)], the glass transition in colloids [Habdas et al. (2004)], as well as fundamental interactions between pairs of colloidal spheres [Crocker (1997); Crocker et al. (2000); Levine and Lubensky (2000)] and entropic forces in binary colloids [Crocker et al. (1999)]. Microrheology has also been proposed as a tool for high-throughput material screening [Breedveld and Pine (2003); Schultz and Furst (2011)].

But to study nonlinear response, the tracers must actively drive the microstructure out of equilibrium. In this active or nonlinear microrheology regime, a constant or oscillatory force is applied to the tracers using optical tweezers or magnetic fields, for example. In such a system, the tracer particles undergo displacements not only due to random thermal 
fluctuations but also due to the external force applied directly to the tracer, or "probe." The dispersion is driven out of equilibrium and, as with macrorheology, dynamic responses such as microviscosity can be determined. Since the probe interrogates the material at the microscale, localized material heterogeneity can be explored.

Most work thus far in nonlinear microrheology has focused on steady dynamic behavior, to establish the relationship between steady-state microstructural mechanics and transport properties, such as the diffusivity, viscosity, and stress [Squires and Brady (2005); Khair and Brady (2006); Zia and Brady (2010, 2012)]. Transient behavior has been studied in the near-equilibrium, linear-response regime-in the microrheological context via its connection to low-amplitude oscillatory probe forcing and the complex modulus [Khair and Brady (2005)]. Such studies show that for very weak forcing, the microstructural response that drives stress (and relaxation) is indistinguishable from equilibrium fluctuations. Previous macrorheological studies of transient behavior in colloidal dispersions also reveal a range of interesting time-dependent phenomena, including temporary stress overshoot behavior, viscoelastic and memory effects such as strain recovery, and the existence of multiple relaxation modes.

Stress formation and relaxation in colloidal dispersions accompanies the storage and loss of microstructural memory. In equilibrium colloidal dispersions, thermal fluctuation of a single particle in a solvent is dissipated back to the solvent via viscous drag [Einstein (1906); Perrin (1909)]. The decay of viscous particle momentum occurs on the order of $10^{-7} \mathrm{~s}$ (for a $0.5-\mu \mathrm{m}$ particle in water); from a particle perspective, memory loss is nearly instantaneous [for a discussion of relaxation on the solvent time scale, see, e.g., Russel (1981); Hocquart and Hinch (1983)]. But a particle diffusing through a dispersion of other particles deforms the suspension, giving rise to stresses that relax on time scales much longer than the individual-particle momentum-relaxation time. The temporal decay of stress (and velocity) fluctuations can be understood from the perspective of linear response theory [Green (1954); Kubo (1966)]: While the shear stress in an equilibrium system is zero on average, thermal fluctuations produce small perturbations in microstructural isotropy, which give rise to temporary shear stresses, which are characterized by the shear stress autocorrelation function, $C_{s}(t)=\left\langle\sigma_{x y}^{\prime}(t) \sigma_{x y}^{\prime}(0)\right\rangle$. Stress relaxation accompanies memory decay. \{The time integral of the stress correlation contributes to the effective suspension viscosity, $\eta \sim \int C_{s}\left(t^{\prime}\right) d t^{\prime}$ [Nägele and Bergenholtz (1998)].\} One may also view this as a connection between viscous dissipation and memory loss. A similar connection between the suspension stress and nonequilibrium fluctuation dissipation has been made by Zia and Brady (2012). Here, we seek to understand the link between the development and relaxation of material stress and microstructural memory by studying the transient viscosity during startup and cessation of microrheological flow.

A material may have multiple relaxation modes which can be probed via lowamplitude oscillatory displacements, for example. The high-frequency limit corresponds to the shortest relaxation time scale of the material; for hard-sphere colloidal dispersions, the stress decays as $t^{-1 / 2}$ at very short times (in the absence of hydrodynamic interactions) [Lionberger and Russel (1994); Brady (1993); Khair and Brady (2005)]. Nonequilibrium transient behavior has also been studied experimentally for sheared dispersions, where it has been shown that multiple mechanisms play a role in suspension stress and viscosity - e.g., hydrodynamic, interparticle, and Brownian forces-and give rise to distinct relaxation processes. For example Mackay and Kaffashi (1995) and Kaffashi et al. (1997) studied the decay of stress immediately after the cessation of imposed strain-rate on a sheared suspension; they found that the hydrodynamic stress decays instantaneously, as it must - the hydrodynamic stress is proportional to the imposed strain-rate, and thus must vanish in the absence of flow. Watanabe et al. (1996b) and Watanabe et al. (1996a) 
analyzed stress development and relaxation in sudden startup and cessation of shearing flow and found both short- and long-time relaxation modes. These studies show that the macroscopic stress relaxes via distinct transport processes, but the microstructural evolution that accompanies this relaxation is not as thoroughly studied.

Although at long times after flow has been initiated a colloidal dispersion behaves as a viscous fluid, at early times during the startup of the flow an "overshoot" in the material stress or viscosity may be observed [Foss (1999)], mirroring the microstructural rearrangements responsible for the transition from elastic to viscous behavior. When the flow is shut off, one would expect the elastic behavior to manifest as creep recovery: Removal of the force should allow the microstructure to return to a previous state.

We explore these connections here by studying dispersion behavior during startup and cessation of a strong flow imposed by forcing a single particle through a suspension. While the problem of steady-state microrheology is well-studied, the transition from equilibrium (frame $a$ in Fig. 1) to steady state (frame $b$ ) and the relaxation back to equilibrium when forcing is shut off (frame $c$ ) is not. To focus on structural aspects of the flow, we shall neglect hydrodynamic interactions (Stokes drag on a particle and the single-particle stresslet are still present, but all higher-order hydrodynamic effects are taken to be negligible), an approximation relevant in many systems, such as dispersions of particles with large electrostatic repulsion lengths or steric repulsion due to, e.g., polymer hairs grafted to the particles' surfaces. Whether one studies stress or velocity fluctuations in a dispersion, the central physical process is the development and subsequent relaxation of the deformed shape of the microstructure. In microrheology, the force/velocity relationship is analogous to the stress/strain-rate relationship in macrorheology. We thus expect both approaches to yield the same qualitative information about structural evolution and relaxation.

The remainder of this paper is set out as follows: A theoretical framework is introduced in Sec. II in which the time-dependent evolution of the microstructure and its connection to the time-dependent viscosity is developed, and the distinction between "constant-force" (CF) and "constant-velocity" (CV) microrheology is reviewed. Next, the transient microstructure is solved analytically using a combination of asymptotic analysis (Secs. III and IV) and numerical methods (Sec. V). In Sec. VI, the results for the transient viscosity measured via Brownian dynamics (BD) simulation are given, for both $\mathrm{CF}$ and $\mathrm{CV}$ regimes. Here, it is shown that the two modes drive qualitatively different startup responses of the microstructure: A stress overshoot is seen in the CV regime. The viscosity at long times after startup is compared to the steady-state theory predictions of

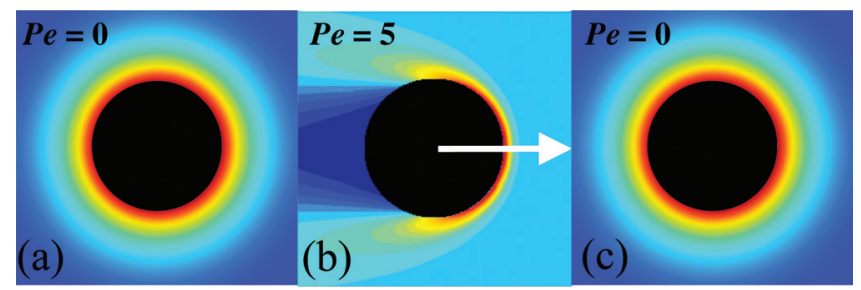

FIG. 1. Contour plot of microstructure around the probe, in the plane passing through the center of the probe. Red and yellow indicate strong and moderate probability accumulation, respectively; dark blue and light blue indicate strong and moderate depletion, respectively. Black is excluded volume around probe. (a) Prior to startup of the flow, the microstructure is at equilibrium. (b) Long after flow startup, the steady-state structure forms; here, for $P e=5$, a probability accumulation layer forms on the upstream face of the probe, and a depletion wake trails it. (c) Long after flow cessation, the Brownian motion of the bath particles restores the equilibrium microstructure. 
Squires and Brady (2005) and to the dynamic simulation of steady-state microrheology of Carpen and Brady (2005) with good agreement. Next, in Sec. V II, it is shown that the elastic energy stored in the deformed microstructure can be partially recovered upon removal of the external force, and a connection is made between the magnitude of this creep recovery and the osmotic compressibility-indicating that the stress may be understood as free-energy storage in the deformed microstructure. An analysis of relaxation modes reveals that pair interactions dominate the relaxation, even for very concentrated suspensions, and the dilute theory is shown to successfully predict behavior up to high volume fractions. Plots of the deformed microstructure captured at key times throughout the evolution of the stress are presented, and it is shown that nearly all stress formation and relaxation occur with the formation and relaxation of a boundary layer-and the majority of structural rearrangement occurs with very little change in stress. The work is also compared to analogous results for sheared suspensions undergoing startup and cessation [Foss (1999)] in Sec. VIII. The investigation is concluded with a discussion of results in Sec. IX.

\section{THEORETICAL FRAMEWORK}

\section{A. Microrheology: Model system and background}

The model system comprises a dispersion of neutrally buoyant colloidal particles of size $b$ immersed in a Newtonian solvent of viscosity $\eta$ and density $\rho$. A Brownian probe particle of size $a$ is driven by an external force through the suspension. The relative strength of fluid inertia to viscous shear defines a Reynolds number, $R e=\rho U a / \eta$, where $U$ is the characteristic probe velocity. Because the probe and bath particles are small, $R e \ll 1$; on the particle time scale, inertia can therefore be neglected and the fluid mechanics are governed by Stokes' equations. The number density of probes $n_{a}$ relative to the number density of bath particles $n_{b}$ is small: $n_{a} / n_{b} \ll 1$. As a probe moves through the bath, it drives the suspension from equilibrium; simultaneously, the Brownian motion of the bath particles acts to recover their equilibrium configuration, giving rise to an entropic restoring force of order $k T / b$, where $k T$ is the thermal energy of the bath. The degree to which the suspension is driven from equilibrium, and hence its effect on probe motion, is determined by the strength of external probe forcing $\boldsymbol{F}^{\text {ext }}$ compared to thermal restoring force $k T / b$, defining a Péclet number: $P e=F^{e x t} /(k T / b)$. This interplay between probe motion and microstructural response gives rise to changes in probe velocity, which can be used to interrogate suspension properties [Squires and Brady (2005); Meyer et al. (2006); Khair and Brady (2006); Wilson et al. (2009); Zia and Brady (2010, 2012)].

In general, the particles interact through hydrodynamic and interparticle forces which may be both short- and long-ranged; the simplest model for the interactive force, which shall be adopted here, is the hard-sphere potential. Thusly defined, the colloids exert no force on each other until their surfaces touch, i.e., when their separation $r=a+b$. At contact, an infinite potential $V(r)$ prevents their overlap:

$$
V(r)= \begin{cases}\infty, & r<a+b \\ 0, & r>a+b\end{cases}
$$

The radii $a$ and $b$ at which the particles exert the hard-sphere force may or may not be the same as their physical or hydrodynamic radii, $a_{h}$ and $b_{h}$, where the no-slip boundary condition is met. Various physical conditions of the colloid or solvent can extend the effective size of the particle beyond the hydrodynamic radius, e.g., steric repulsion or an ionic screening layer. The particles then repel each other at their extended or 


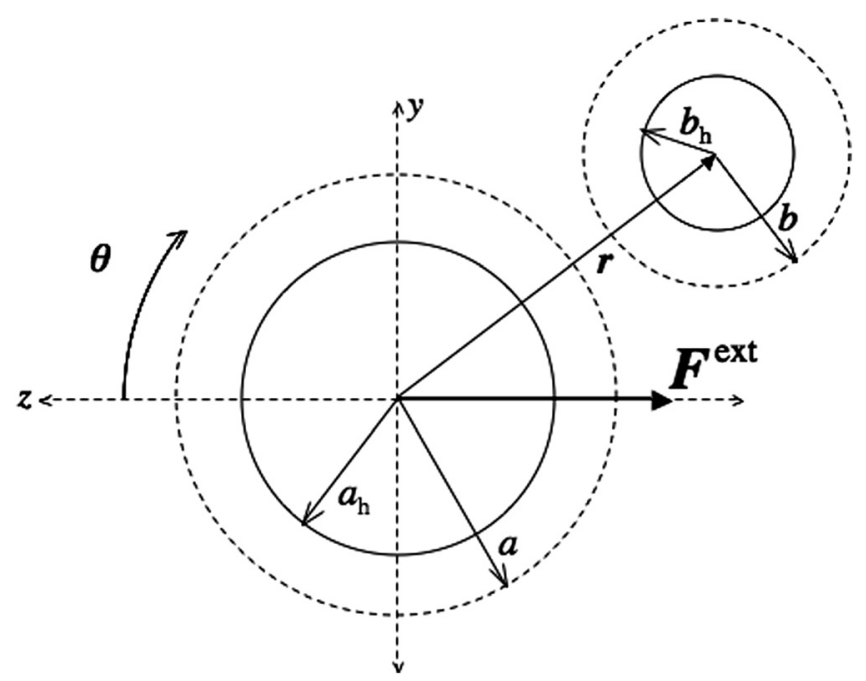

FIG. 2. Pair interaction for probe and bath particle.

"thermodynamic" radii, $a$ and $b$ (see Fig. 2). This approach forms the foundation of the excluded annulus model of Morris and Brady (1996), in which the ratio of the two radii ( $\lambda_{a}=a / a_{h}$ and $\left.\lambda_{b}=b / b_{h}\right)$ can be modulated to account for the relative importance of hydrodynamic-to-interparticle forces. When $\lambda_{a}, \lambda_{b} \gtrsim O(1)$, the particles are able to approach each other closely enough to experience (long-range) hydrodynamic interactions. For $\lambda_{a}, \lambda_{b} \rightarrow 1$, lubrication interactions also become important. In the limit $\lambda_{a}, \lambda_{b} \gg 1$, strong interparticle repulsion keeps the particles sufficiently separated that hydrodynamic interactions are negligibly weak. Although for this study attention is focused on the limit of no hydrodynamic interactions, $\lambda_{a}, \lambda_{b} \rightarrow \infty$, the model captures the essential features of dispersive and dissipative processes while allowing clarity in the exposition of the behavior.

As the probe particle moves through the suspension, it must push neighboring particles out of its way; at steady state, a buildup of background particle concentration forms in front of the advancing probe and a deficit or wake trails it. Brownian diffusion of the bath particles acts to restore isotropy, but as $P e$ is increased, advection wins the competition and the microstructural deformation becomes highly anisotropic. Figure 3 gives an illustration of the steady-state deformed microstructure at several values of the Péclet number, where $P e$ increases from left to right in the frames. The microstructure hinders the probe, slowing its motion. Squires and Brady (2005) and Khair and Brady (2006) interpreted the mean-speed reduction as a viscous drag of the bath and defined an effective viscosity $\eta^{\text {eff }}$ via application of Stokes' drag law to the average velocity $\langle\mathbf{U}\rangle$ of the probe

$$
\mathbf{F}^{e x t}=6 \pi \eta^{e f f} a\langle\mathbf{U}\rangle
$$

The effective steady viscosity is then given by

$$
\frac{\eta^{e f f}}{\eta}=\frac{F^{e x t}}{6 \pi \eta a\langle U\rangle}
$$

where $\langle U\rangle=-\langle\mathbf{U}\rangle \cdot \mathbf{F}^{\text {ext }} / F^{\text {ext }}$. The effective viscosity may be written as 


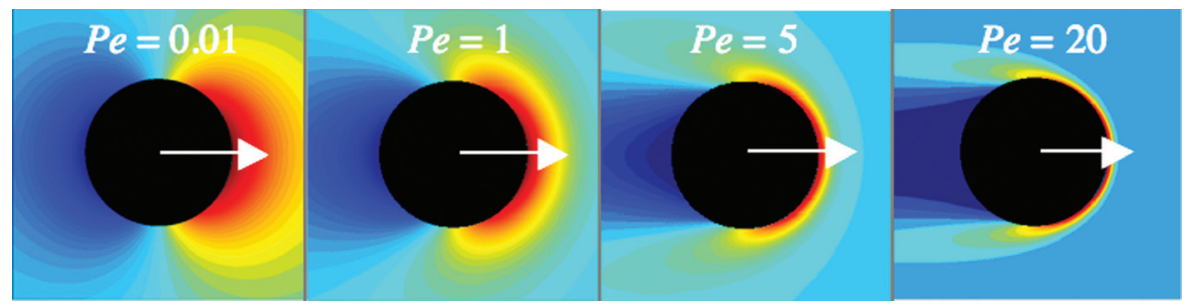

FIG. 3. Theoretical predictions for the deformed microstructure around a moving probe particle in the absence of hydrodynamic interactions at the pair level. The test particle is moving to the right and there is a build-up of background particle density in front (red) of the probe and a deficit (dark blue) in the trailing wake [Squires and Brady (2005)].

$$
\frac{\eta^{e f f}}{\eta}=1+\frac{\eta^{\text {micro }}}{\eta}
$$

thus defining the microviscosity, $\eta^{\text {micro }} / \eta$, as the viscous drag of the particle microstructure-above and beyond the solvent viscosity. In general it includes hydrodynamic, interparticle, and Brownian contributions: $\eta^{\text {micro }}=\eta^{\text {micro }, H}+\eta^{\text {micro }, P}+\eta^{\text {micro }, B}$.

In the limit of no hydrodynamic interactions $\left(\lambda_{a}, \lambda_{b} \gg 1\right)$, the microviscosity is the average hindrance of the microstructure due to collisions between the probe and the background bath particles. For a hard-sphere potential, disturbances to probe velocity due to interparticle forces occur only at contact between probe and bath particle; the contribution to probe velocity due to collisions with the bath particles, $\left\langle\mathbf{U}^{P}\right\rangle$, is thus given by [Squires and Brady (2005); Khair and Brady (2006)]

$$
\left\langle\mathbf{U}^{P}\right\rangle=-\frac{F^{e x t}}{6 \pi \eta a} \frac{3 \phi_{b}}{4 \pi} P e^{-1} \int \mathbf{n} g(\mathbf{r}) d \Omega
$$

where $g(\mathbf{r})$ is the probe-bath pair-distribution function and $d \Omega$ is the element of solid angle. Here, $\phi_{b}=4 \pi b^{3} n_{b} / 3$ is the volume fraction of bath particles. Combining Eqs. (2), (4), and (5) gives the microviscosity due to hard-sphere collisions, in the limit of no hydrodynamic interactions between particles. Following Squires and Brady (2005), the expression for the microviscosity without hydrodynamic interactions can be written without approximation in the time-dependent form

$$
\left.\frac{\eta^{\text {micro }}(t ; P e)}{\eta}=-\frac{3}{4 \pi} P e^{-1}\left(1+\frac{a}{b}\right)^{2} \phi_{b} \hat{\mathbf{u}} \cdot \int_{r=1} \mathbf{n} g(\mathbf{r}, t ; P e)\right) d \Omega .
$$

Here, the microstructure and hence the viscosity are evolving in time $t$, and $\hat{\mathbf{u}}$ is the unit vector antiparallel to the line of the externally applied force. Lengths have been made dimensionless as $r \sim(a+b)$. The probe-bath pair-distribution function $g(\mathbf{r}, t ; P e)$ is now a function of time. In order to determine how the viscosity evolves from equilibrium to steady state, the time-dependent Smoluchowski equation must be solved in order to obtain $g(\mathbf{r}, t ; P e)$.

To understand the evolution and relaxation of the stress in the dispersion, the important quantity is the reduction in mean probe velocity, $\left\langle\mathbf{U}^{P}\right\rangle$, due to collisions with the other background particles in the dispersion. This is what is determined in both the theory and in Brownian dynamics simulation in the following sections and is what one would measure in an experiment. From the reduction in the speed, one can define the effective 
viscosity, Eq. (3); the specific nomenclature "microviscosity," $\eta^{\text {micro }}$, corresponds to the particle contribution to the effective viscosity.

\section{B. Transient microstructure}

The probability distribution of the bath particles is governed by a Smoluchowski equation, which in the dilute-pair limit is given by $\partial g / \partial t+\nabla \cdot \mathbf{j}=0$, where $\mathbf{j}$ is the probability flux of a particle relative to the probe. Particles move due to advection with the mean flow and due to Brownian diffusion, giving a total flux $\mathbf{j}=\mathbf{U} g-\mathbf{D}_{r} \cdot \nabla g$, where we have defined the relative Brownian diffusivity and the relative velocity between the probe and a bath particle, $\mathbf{D}_{r}=D_{r} \mathbf{I} \equiv\left(D_{a}+D_{b}\right) \mathbf{I}$ and $U \equiv U_{b}-U_{a}$. Here, $\mathbf{I}$ is the identity tensor. For a suspension of hard spheres in the absence of hydrodynamic interactions, the pairSmoluchwoski equation becomes

$$
\frac{\partial g}{\partial t}+\mathbf{U} \cdot \nabla g=D_{r} \nabla^{2} g
$$

The strength of the external forcing compared to the restoring entropic force defines the Péclet number

$$
P e=\frac{U^{0}(a+b)}{D_{r}}
$$

where the length scale is set by $r \sim(a+b)$ and the characteristic velocity $U^{0}=F^{e x t} / 6 \pi \eta a$ is set by the Stokes velocity of an isolated probe. Thus, the evolution of the microstructure is given by

$$
\frac{\partial g}{\partial t}+P e \hat{\mathbf{u}} \cdot \nabla g=\nabla^{2} g
$$

where all quantities are dimensionless, and time has been scaled diffusively, $t \sim(a+b)^{2} / D_{r}$. As noted above, $\hat{\mathbf{u}}$ is the unit vector antiparallel to probe forcing, because in a frame of reference moving with the probe, the relative velocity of the bath is opposite the direction of the applied external force.

Because changes in the microstructure are of interest, it is useful to consider the microstructural perturbation $f$ defined by $g(\mathbf{r}, t ; P e)=1+f(\mathbf{r}, t ; P e)$. The hard spheres permit no flux at contact, and there is no long-range order in the suspension. For flow startup, the sudden onset of forcing appears as an advective term at $t=0$ in the no-flux condition. The governing equations for the startup regime are

$$
\begin{aligned}
\frac{\partial f}{\partial t}+P e \hat{\mathbf{u}} \cdot \nabla f & =\nabla^{2} f, \\
\mathbf{n} \cdot \nabla f & =P e \hat{\mathbf{u}} \cdot \mathbf{n}(f+1) H(t) \quad \text { at } \quad r=1, \\
f & \sim 0 \quad \text { as } \quad r \rightarrow \infty \\
f(\mathbf{r}, t) & =0 \quad \text { at } \quad t=0 .
\end{aligned}
$$

Here, $H(t)$ is the Heaviside function which turns on the flow, and the microstructure is initially undisturbed. The Péclet number is set by the velocity just after the flow is turned on.

When the forcing is removed, the advective term vanishes, giving the governing equations after flow cessation as 


$$
\begin{aligned}
\frac{\partial f}{\partial t} & =\nabla^{2} f, \\
\mathbf{n} \cdot \nabla f & =0 \quad \text { at } \quad r=1, \\
f & \sim 0 \quad \text { as } \quad r \rightarrow \infty, \\
f(\mathbf{r}, t) & =f^{S S}(\mathbf{r} ; P e) \quad \text { at } \quad t=0 .
\end{aligned}
$$

The diffusive relaxation is forced by the initially deformed microstructure-the steadystate microstructure $f^{S S}(\mathbf{r} ; P e)$, prior to removal of the external force. The Péclet number is set by the value of the velocity just prior to shutoff of the flow.

\section{Constant external force versus constant external velocity}

Active microrheology experiments are carried out by two main approaches: Driving the probe with a constant external force-e.g., with magnetic tweezers [Habdas et al. (2004)] — or holding the probe fixed in an optical trap and moving the bath past it at a constant velocity - e.g., with laser tweezers [Meyer et al. (2006)]. ${ }^{1}$ The constant-external force $(\mathrm{CF})$ case corresponds to a "mobility" formulation of the hydrodynamic problemthe force is prescribed, the resultant velocity fluctuates, and as a result, the probe experiences a collision-induced diffusive spread of its trajectory [Zia and Brady (2010)]. In the constant-imposed velocity (CV) case, the velocity is fixed while the force fluctuates-a "resistance" formulation. The difference leads to important dynamical consequences for probe motion and microstructural evolution. Since probe velocity can fluctuate in CF mode, the probe can adjust its speed as it enters a region of higher density, whereas in CV mode, the probe's fixed velocity means it must "bulldoze" through the suspension regardless of fluctuations in local density. The result is a more dense accumulation of particles in the CV boundary layer and a higher effective viscosity; Squires and Brady (2005) showed that the viscosity in CV mode should be twice the CF-mode viscosity for dilute dispersions. This factor of 2 emerges naturally from the scaling of the diffusivity; recall from Eq. (8) that the Péclet number is defined as $P e=U(a+b) / D_{r}$, where $D_{r}$ is the appropriate diffusive scaling. In CF mode, the proper diffusive scaling is the relative diffusivity $D_{F}$ between a pair of particles: $D_{F}=D_{a}+D_{b}=(k T / 6 \pi \eta)(1 / a+1 / b)$, where $D_{a}=k T / 6 \pi \eta a$ and $D_{b}=k T / 6 \pi \eta b$ are the isolated diffusivities of a probe and a bath particle, respectively, and for brevity, the subscript $h$ has been dropped from the radii $a$ and $b$. But in $\mathrm{CV}$ mode, since the probe cannot diffuse, the proper diffusive scaling $D_{U}$ is that of the bath particles, as only they diffuse: $D_{U}=D_{b}=k T / 6 \pi \eta b$. For a probe-to-bath-particle size ratio $a / b=1$, we have that $D_{F}=2 D_{U}$, with the inverse ratio for the effective viscosity (in the limit of no hydrodynamic interactions). Carpen and Brady (2005) evaluated this prediction via Brownian dynamics simulation of steady-state microrheology and found agreement between simulation and theory for all bath-particle volume fractions $\phi_{b}$; the result is not restricted to dilute baths, although there is a slight quantitative difference at higher concentrations. In this study, we also investigate by dynamic simulation the long-time startup behavior in transient microrheology of the two modes. Our results are compared in later sections to the steady-state values reported by Squires and Brady and Carpen and Brady.

\footnotetext{
${ }^{1}$ The idea of a constant force or a constant velocity is an approximation, the accuracy of which is dictated by the uniformity of the applied field or the "stiffness" of the optical trap (which is controlled via adjustments of laser power). Such approximations can be made quite accurate [Habdas et al. (2004); Meyer et al. (2006)].
} 


\section{ANALYTICAL SOLUTION: FLOW STARTUP}

In this section, the analytical solution for the constant-force $(\mathrm{CF})$ mode of transient microrheology is obtained in the limits of weak and strong external forcing. In the startup regime, Eq. (10) are solved with the Péclet number defined for the CF mode in Sec. II C. Constant-velocity $(\mathrm{CV})$ forcing results follow, by simply replacing $D_{F}$ with $D_{U}$. All analytical results are for dilute systems, $\phi_{b} \ll 1$.

\section{A. Low-Pe limit}

In steady (nonlinear) microrheology, the perturbation to the microstructure due to weak probe forcing is $f(\mathbf{r} ; P e)=-P e \hat{\mathbf{u}} \cdot \mathbf{n} h(r)$, where $r$ is the separation between the probe and a bath particle, $h \sim 1 / r^{2}$, and for the steady case, $h$ is independent of time [Squires and Brady (2005)]. Here, $\mathbf{n}$ is the unit surface normal pointing from the probe to the bath particles. The time-dependent structure has the form $f(\mathbf{r}, t ; P e)=-P e \hat{\mathbf{u}} \cdot \mathbf{n} e^{i \alpha t} h(r, t)$, where $\alpha=\omega(a+b)^{2} / D_{r}$ is the dimensionless frequency [Khair and Brady (2005)]. It is convenient to analyze the evolution of the microstructure in frequency space. Following the approach of Brady (1994) and Khair and Brady (2005), we insert the proposed form of the solution into Eq. (10) and take the Laplace transform with respect to time to give

$$
\begin{aligned}
\frac{1}{r^{2}} \frac{d}{d r}\left(r^{2} \frac{d \tilde{h}}{d r}\right)-\frac{2}{r^{2}} \tilde{h}-i \alpha \tilde{h} & =0, \\
\frac{d \tilde{h}}{d r}+\frac{1}{2} \sqrt{\frac{\pi}{2}}\left[\frac{1}{i \alpha}-\pi \delta(\alpha)\right]=0 \quad \text { at } \quad r & =1, \\
& \tilde{h} \sim 0 \quad \text { as } \quad r \rightarrow \infty
\end{aligned}
$$

Here, $\alpha$ is the variable transforming time to frequency space, the tilde $\sim$ indicates a transformed quantity, and $i$ is the imaginary unit. With the substitution of variable $z=r(i \alpha)^{1 / 2}$, Eq. (12) is the modified spherical Bessel equation with solution [Brady (1994)]

$$
\tilde{h}\left(r, z_{0}\right)=\frac{2}{r^{2}} \exp \left[z_{0}(1-r)\right]\left[\frac{1+z_{0} r / 2}{1+z_{0}+z_{0}^{2} / 2}\right]\left(\pi \delta\left(-i z_{0}^{2}\right)+\frac{1}{z_{0}^{2}}\right),
$$

where $z_{0}=2(i \alpha)^{1 / 2}$. To compute the frequency-space microviscosity, the microstructure at contact $(r=1)$ is required:

$$
\frac{\eta^{\text {micro }}(t)}{\eta}=-4 \mathcal{L}^{-1}\left[2 \frac{1+\sqrt{s} / 2}{1+\sqrt{s}+s / 2}\right]\left(\pi \delta(i s)+\frac{1}{s}\right)
$$

when $s=z_{0}^{2}$. The inverse transform in Eq. (14) can be obtained numerically in MATHEMATICA [Valko and Abate (2002)] and the transient microviscosity is plotted in Fig. 4. The microviscosity increases in time very rapidly after the onset of probe forcing as

$$
\frac{\eta^{\text {micro }}(t)}{\eta} \sim t^{1 / 2} \quad \text { as } \quad t \rightarrow 0
$$

which follows from the limit of Eq. (14) as $s \rightarrow \infty$. As $t \rightarrow \infty$, the viscosity asymptotes algebraically to a steady value as $\eta^{\text {micro }} / \eta \sim 2-t^{-3 / 2}$, as is found in the zero-frequency limit for small-amplitude, linear oscillatory motion [Khair and Brady (2005)], and the weakly nonlinear disturbance of diffusion in suspensions [Brady (1994)]. The long-time 


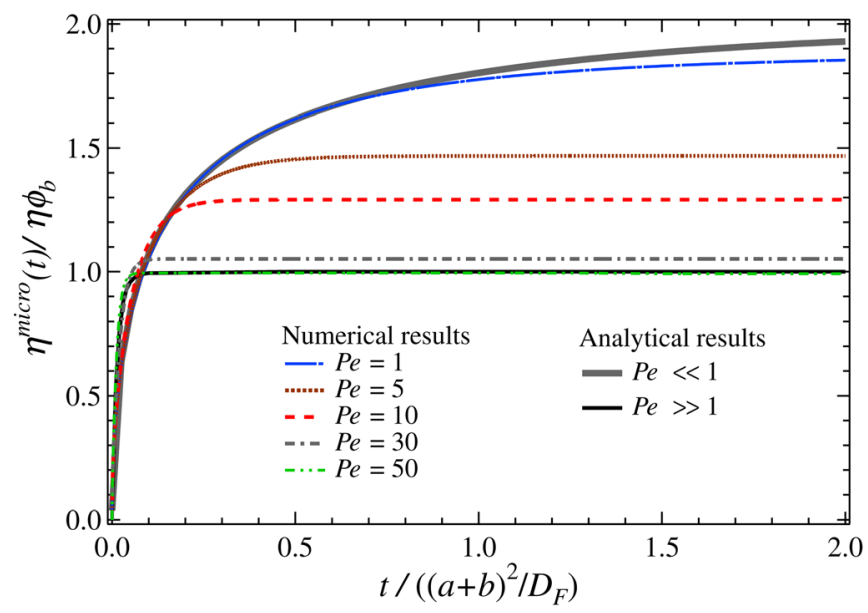

FIG. 4. Startup regime, constant external force: Solid grey and black lines are analytical solutions for $P e \ll 1$ [Eq. (14)] and $P e \gg 1$ [Eq. (18)], respectively. Broken lines represent numerical solution of Eq. (26). The initial increase in the viscosity is steep and appears to be independent of $P e$. As $t /\left((a+b)^{2} / D_{F}\right) \rightarrow \infty$, the microstructure reaches steady state and the viscosity asymptotes to its steady value.

limit is compared in Table I to the small-Pe steady-state values computed by Squires and Brady (2005); the two agree.

\section{B. High-Pe limit}

For strong probe forcing $(P e \gg 1)$, the steady-state shape of the microstructure in front of the probe is deformed into two distinct regions: An outer region in which advection dominates diffusion and the microstructure is undisturbed; and an inner region, a $1 / P e$-thin boundary layer that forms on the upstream face of the probe where diffusion balances unsteady advection because the relative flux at contact is zero. Upon sudden startup, a diffusive step of the probe is executed first; the boundary layer then begins to form on the time scale of the flow, $t \sim a / U \sim \tau_{D} / P e$, where the time scale for Brownian diffusion is $\tau_{D}=(a+b)^{2} / D_{r}$. The first emergence of the boundary layer is thus nearly instantaneous after the first step as $P e \rightarrow \infty$. Although complete evolution of the boundary layer may take a several diffusive time steps, its initial appearance sets the mechanics of structural evolution. Here, we shall proceed with the transient analysis by assuming the presence of a boundary layer at all times.

Inside the boundary layer, a coordinate rescaling $R=(r-1) P e \sim O(1)$ preserves the diffusive term, properly reflecting the physics of the inner region and allowing satisfaction of the no-flux condition at contact. The appropriate scaling for time is the characteristic diffusive time, but since probe diffusion takes place inside the boundary layer of

TABLE I. Comparison of the transient microviscosity at long times after startup obtained in this study [numerical solution of Eq. (26)], to the steady-state value obtained from solving the steady microrheology problem [Squires and Brady (2005); Khair and Brady (2006); Zia and Brady (2010)].

\begin{tabular}{lcc}
\hline \hline$P e$ & $\eta_{S S}^{\text {micro }} / \eta \phi_{b}$ & $\eta^{\text {micro }}(t \rightarrow \infty) / \eta \phi_{b}$ \\
\hline 1 & 1.88 & 1.9 \\
5 & 1.48 & 1.48 \\
10 & 1.32 & 1.29 \\
30 & 1.14 & 1.05 \\
50 & 1.10 & 1.00 \\
\hline \hline
\end{tabular}


thickness $\delta \sim(a+b) / P e$, time is scaled as $\tau=t /\left(\delta^{2} / D_{r}\right)=t / P e^{2}$. To proceed formally with the boundary-layer solution, a perturbation expansion of $f(\mathbf{r}, t ; P e)$ in $P e$ is performed following the steady-state analysis of Khair and Brady (2006); in the steady case, Khair and Brady solve the first and second order perturbation to the microstructure, yielding a coupled pair of ordinary differential equations. In the present time-dependent case, the first two orders in perturbation yield two coupled partial differential equations describing the spatiotemporal evolution. Alternatively, in a more informal approach, one may argue physically that one need to retain only the leading order in diffusive, advective, and temporal behavior. The two approaches for obtaining the microstructure agree, and the latter technique is presented here. Retaining the leading order terms, one may discard terms of order $P e^{-2}$ and smaller, which then gives

$$
\frac{\partial f}{\partial \tau}+\frac{\partial^{2} f}{\partial R^{2}}+\left(2 P e^{-1}-\cos \theta\right) \frac{\partial f}{\partial R}-P e^{-1} \sin \theta \frac{\partial f}{\partial \theta}=0,
$$

for the microstructure in the upstream region, with the corresponding restriction $\cos \theta \leq 0$; the polar angle $\theta$ is as defined in Fig. 2. All quantities are dimensionless. As $P e \rightarrow \infty$, radial gradients are much stronger than angular gradients in the deformed microstructure and, to first approximation, the latter may be neglected. For large $P e$, the resulting governing equations become, after taking a Laplace transform,

$$
\begin{aligned}
\frac{d^{2} \tilde{f}}{d R^{2}}+\left[2 P e^{-1}-\cos \theta\right] \frac{d \tilde{f}}{d R}-s \tilde{f} & =0, \\
\frac{d \tilde{f}}{d R}-\cos \theta\left(\tilde{f}+\frac{1}{s}\right)=0 \quad \text { at } \quad R & =0, \\
\tilde{f} & \sim 0 \quad \text { as } \quad R \rightarrow \infty .
\end{aligned}
$$

The $O\left(P e^{-1}\right)$ term must be retained in order to satisfy the boundary condition as $R \rightarrow \infty$ [Brady and Morris (1997)]. This initial value problem has an exact solution; upon inverting, one obtains for the microstructural disturbance at contact

$$
\begin{aligned}
f(1, \mu, \tau)= & \frac{1}{4}\left[(P e \mu-2) \operatorname{Erf}\left(\frac{\sqrt{t}(2-P e \mu)}{2 P e}\right)+(P e \mu+2)\right. \\
& \left.\times\left(\exp \left[\frac{2 t \mu}{P e}\right] \operatorname{Erfc}\left(\frac{\sqrt{t}(2+P e \mu)}{2 P e}\right)-1\right)\right],
\end{aligned}
$$

where for compactness, we have defined $\mu \equiv \cos \theta$ and $\tau \equiv t / P e^{2}$. This expression is then inserted into Eq. (6) and integrated numerically to obtain the transient microviscosity. As seen in Fig. 4, the microviscosity increases very rapidly after the onset of forcing. The early-time behavior can be seen more clearly in the log-log plot in Fig. 5: The microviscosity initially grows with the square root of time. Importantly, the early-time behavior of the transient microviscosity is the same regardless of the strength of external forcing. This is because the first step executed by the probe is diffusive and scales as $\sim \sqrt{\Delta t}$, which is larger than the $O(\Delta t)$-size advective step at short times. Thus, the very short-time (infinite-frequency) viscosity corresponds to the short-time self-diffusive behavior of the probe. At intermediate times, the exponential and constant terms dominate, and the microviscosity asymptotes to a time-independent steady value as $\sim 1-e^{-t}$. The long-time microviscosity is compared in Table I to the high- $P e$ steady-state values computed by Squires and Brady (2005); the two agree. 


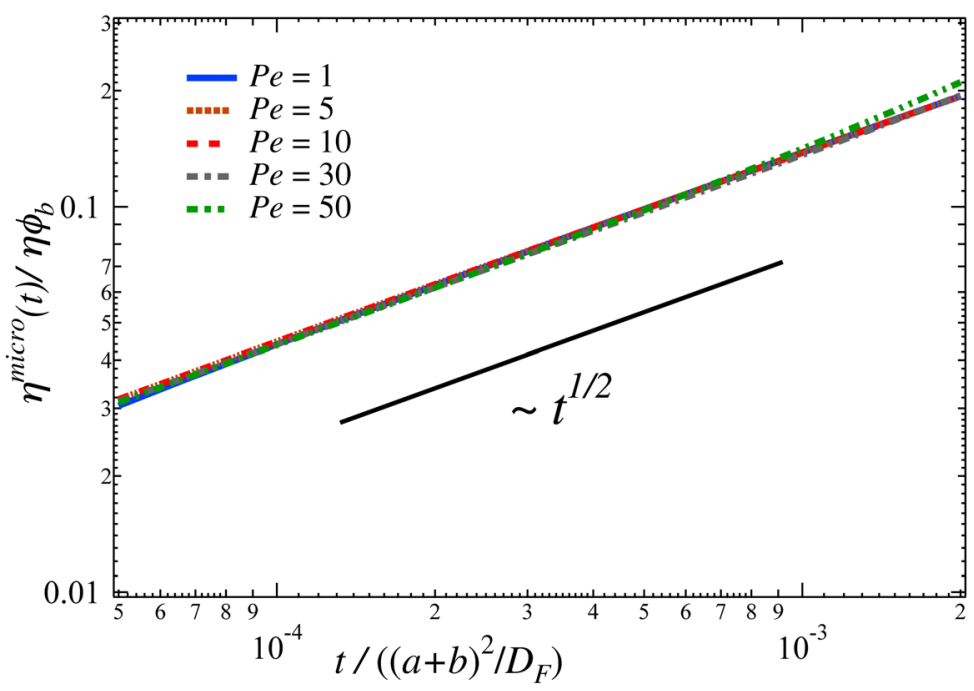

FIG. 5. Startup regime, constant external force, at early times after startup [numerical solution of Eq. (26)]. Microviscosity grows as $t^{1 / 2}$ regardless of $P e$.

\section{ANALYTICAL SOLUTION: FLOW CESSATION}

In this section, the microstructural evolution after force cessation is obtained in two limits: The relaxation of a weakly deformed and a strongly deformed microstructure. Equation (11) are solved for $P e \ll 1$ and $P e \gg 1$, respectively, where the Péclet number corresponds to the pre-cessation value. As with the startup regime considered above, the CF mode is studied, using the appropriate Péclet number (cf. Sec. II C).

\section{A. Low-Pe limit}

In the limit $P e \ll 1$, the steady-state linear response of the microstructure is given by $f_{S S}(\mathbf{r} ; P e)=-P e \cos \theta / 2 r^{2}$ [Squires and Brady (2005)]. When the probe force is shut off, this initial dipolar structure sets the subsequent relaxation behavior, which is governed by Eq. (11), whose Laplace transform reads

$$
\begin{aligned}
\nabla^{2} \tilde{f}-s \tilde{f} & =f_{S S}(\mathbf{r} ; P e), \\
\frac{d \tilde{f}}{d r} & =0 \quad \text { at } \quad r=1, \\
\tilde{f} & \sim 0 \quad \text { as } \quad r \rightarrow \infty
\end{aligned}
$$

where time is transformed to the frequency variable $s$, and the tilde $\sim$ indicates a transformed quantity. The particular solution is given by $\tilde{f}^{P}=-f_{S S}(\mathbf{r} ; P e) / s$. The corresponding homogenous equation is a Helmholtz equation; a solution by separation of variables into angular and radial coordinate is straightforward, where the angular solutions are Legendre polynomials $P_{n}(\cos \theta)$. The radial differential equation is Bessel's equation; its solutions are the modified spherical Bessel functions of the third kind, $h_{n}(\sqrt{s} r)=\sqrt{\pi / 2 \sqrt{s} r} K_{n+1 / 2}(\sqrt{s} r)$ [Abramowitz and Stegun (1964)]. The boundary condition at contact eliminates all Legendre modes except the first, giving the perturbed microstructure at contact 


$$
\tilde{f}(1, \theta, s)=\frac{1}{2} P e\left(\frac{1}{2+2 \sqrt{s}+s}\right) \cos \theta .
$$

The microstructure can be inverted numerically to obtain the microviscosity:

$$
\frac{\eta^{\text {micro }}(t)}{\eta}=2 \mathcal{L}^{-1}\left[\frac{1}{2+2 \sqrt{s}+s}\right] .
$$

The result is plotted in Fig. 6. At least two relaxation modes are seen: An initially rapid decay, followed by a slower, long-time decay.

\section{B. High-Pe limit}

Prior to shutoff of strong probe forcing, the well-defined boundary layer sets the dynamics of probe motion and of the microviscosity. The appropriate time scale is now diffusive in the boundary layer, $\tau \sim t / P e^{2}$. A radial coordinate rescaling $R \sim P e(r-1)$ (cf. Sec. III B) preserves the diffusive term during relaxation. A perturbation expansion of the deformed microstructure in inverse powers of $P e$ inside the boundary layer is appropriate: $f(R, \theta ; P e)=P e f_{0}+f_{1}+P e^{-1} f_{2}+\cdots$ To leading order in $P e$ and with this rescaling in $R$, the dimensionless governing equations become, upon taking a Laplace transform,

$$
\begin{aligned}
\frac{d^{2} \tilde{f}_{0}}{d R^{2}}-s \tilde{f}_{0} & =f^{S S}(R ; P e), \\
\frac{d \tilde{f}_{0}}{d R} & =0 \quad \text { at } \quad R=0, \\
\tilde{f}_{0} & \sim 0 \quad \text { as } \quad R \rightarrow \infty,
\end{aligned}
$$

where $f^{S S}(R ; P e \rightarrow \infty)=-P e \cos \theta e^{R \cos \theta}$ is the steady-state microstructure [Squires and Brady (2005)] prior to flow shutoff and $\cos \theta \leq 0$ (cf. Fig. 2). The solution can be inverted analytically and at contact is given by

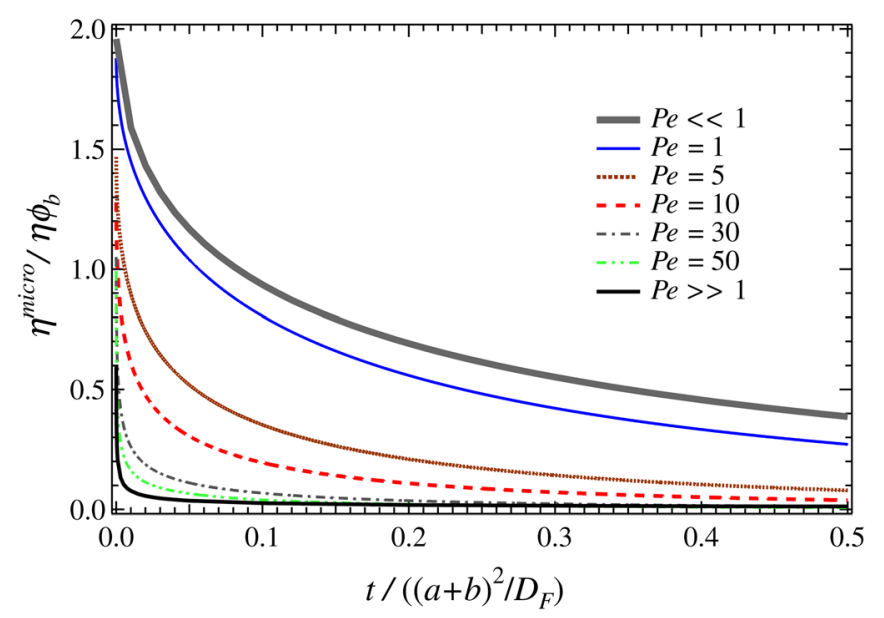

FIG. 6. Flow cessation: The solid grey and black lines are the analytical solution for $P e \ll 1$ [Eq. (21)] and $P e \gg 1$ [Eq. (24)], respectively. Broken lines are numerical solutions of Eq. (27). Viscosity drops rapidly from its steady-state value. The rate at which the viscosity and stress relax increases with increasing $P e$. The structure continues to relax more slowly at long times. 


$$
f_{0}(1, \theta, \tau)=-f^{S S}\left[\exp \left(\tau \cos ^{2} \theta\right) \operatorname{Erfc}(\sqrt{\tau} \cos \theta)\right] .
$$

Insertion into Eq. (6) gives for the microviscosity

$$
\frac{\eta^{\text {micro }}(\tau)}{\eta}=\frac{1}{\tau}\left[e^{\tau} \operatorname{Erfc}(\sqrt{\tau})+2 \sqrt{\frac{\tau}{\pi}}-1\right] .
$$

The results are plotted in Fig. 6, showing an initially rapid decay of the stress at very short times followed by a slower long-time decay, indicating at least two relaxation modes. At early times, the stress decays with the square-root of time:

$$
\frac{\eta^{\text {micro }}(\tau)}{\eta} \sim \frac{1}{\tau^{1 / 2}}
$$

indicating that the initial period of structural relaxation is that of one-dimensional diffusion in the thin, locally planar boundary layer (see Fig. 7). The majority of the stress relaxation thus occurs as the result of boundary-layer diffusion. At longer times, the exponential character of the decay dominates; here, memory of the initial disturbance is lost and, as equilibrium is approached, the probe's relaxation is indistinguishable from the behavior for small Pe obtained in Sec. IV A.

\section{NUMERICAL SOLUTION FOR ARBITRARY Pe}

To capture the full behavior of the transient microstructure over a range of $P e$, a numerical solution of the full Smoluchowski equations (10) and (11) is required. Using a second-order finite difference scheme, radial and angular gradients are discretized onto a grid in both space and time. In order to properly capture the spatial gradients both near and far from particle contact, the radial coordinate is rescaled with the stretched coordinate $R=P e(r-1)$; this rescaling captures near- and far-field behavior over a wide range of $P e$ with an appropriate distribution of discretized grid points (discussed below). Because the flow is axisymmetric about the line of external forcing, derivatives of $g(\mathbf{r})$ in

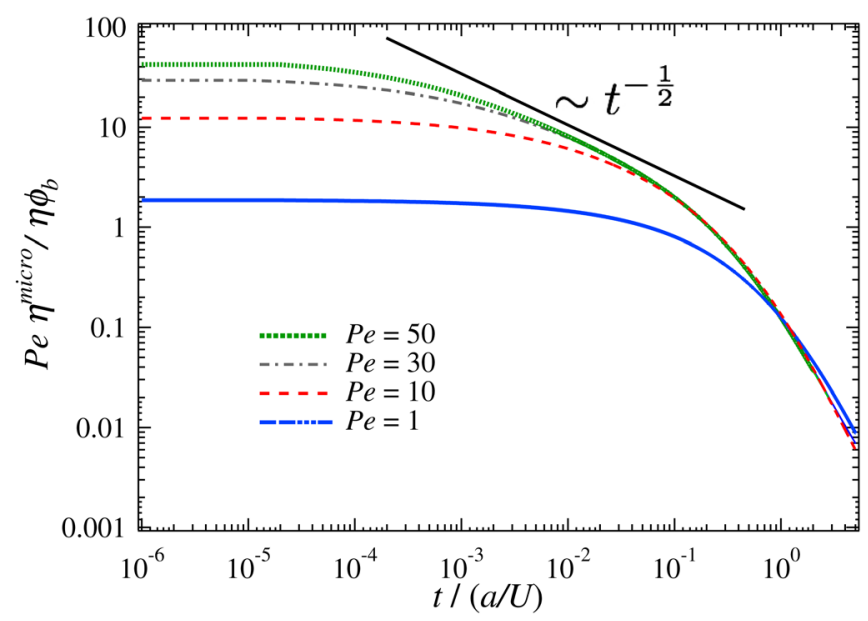

FIG. 7. Flow cessation: Numerical solution of Eq. (27). Viscosity plotted on advective time scale, where $U=F^{e x t} / 6 \pi \eta a$ was the velocity of the probe prior to shutoff. All lines collapse together at long times, indicating the loss of memory of the initial condition. 
the azimuthal angle are zero. Defining $\rho \equiv 1+R / P e$, we have for the governing equations at startup

$$
\begin{aligned}
P e^{-2} \frac{\partial f}{\partial t} & =\frac{\partial^{2} f}{\partial \rho^{2}}+P e^{-1} \frac{2}{\rho} \frac{\partial f}{\partial \rho}+\frac{P e^{-2}}{\rho^{2} \sin \theta} \frac{\partial}{\partial \theta}\left(\sin \theta \frac{\partial f}{\partial \theta}\right)-\cos \theta \frac{\partial f}{\partial \rho}+P e^{-1} \frac{\sin \theta}{\rho} \frac{\partial f}{\partial \theta}, \\
\frac{\partial f}{\partial \rho} & =\cos \theta(f+1) \quad \text { at } \quad \rho=0 \\
f & =0 \quad \text { as } \quad \rho \rightarrow \infty \\
f & =0 \quad \text { at } \quad t=0 .
\end{aligned}
$$

For flow cessation, the time-dependent diffusion equation (11) becomes

$$
\begin{aligned}
P e^{-2} \frac{\partial f}{\partial t} & =\frac{\partial^{2} f}{\partial \rho^{2}}+P e^{-1} \frac{2}{\rho} \frac{\partial f}{\partial \rho}+P e^{-2} \frac{1}{\rho^{2} \sin \theta} \frac{\partial}{\partial \theta}\left(\sin \theta \frac{\partial f}{\partial \theta}\right), \\
\frac{\partial f}{\partial \rho} & =0 \quad \text { at } \quad \rho=0 \\
f & =0 \quad \text { as } \quad \rho \rightarrow \infty, \\
f & =f^{S S}(\rho, \theta ; P e) \quad \text { at } \quad t=0 .
\end{aligned}
$$

Once the boundary layer forms, the radial gradients in the microstructure become increasingly confined to the boundary layer. As $P e$ continues to grow and the boundary layer thins, a grid point concentration function that varies with $P e$ increases the density of grid points close to contact yet retains sufficient resolution far from the probe to capture the physics throughout the upstream domain [Bergenholtz et al. (2002)]. The difference coefficients and operators for both radial and angular directions are compactly arranged into firstand second-order sparse matrices, and the discretized partial differential equation takes on the form

$$
\mathbf{M} \cdot \boldsymbol{f}=\dot{\boldsymbol{f}},
$$

where $\boldsymbol{f}$ is the solution array for all grid points, $\dot{\boldsymbol{f}} \equiv \partial \boldsymbol{f} / \partial t$, time is scaled on the boundary layer thickness, and $\mathbf{M}$ is the super-matrix containing the coefficient and finite-difference matrices that operate on the solution $f$. The solution for the homogeneous (i.e., time-independent) pair-distribution microstructure $f^{S S}(\mathbf{r} ; P e)$ is obtained by solving Eq. (28) with the right-hand side equal to zero, where the boundary condition at contact results in a nontrivial solution. The homogeneous problem was solved utilizing MATLAB and a LAPACK solver. To obtain the transient microstructure over the full range of $P e$ during startup and cessation, we recognize that each row in Eq. (28) is a linear ordinary differential equation in time. The method of lines is used to solve this system of initial value problems and is implemented using the ODE45 solver of MATLAB.

\section{A. Startup}

Figure 4 gives a plot of the transient microviscosity for $0 \leq t \leq 2$ for a probe-to-bath size ratio of unity, for several values of $P e$. The initial condition at $t=0$ is the equilibrium microstructure, $f=0$; time has been scaled diffusively. As can be seen in the figure, the structure initially evolves at the same rate regardless of $P e$.

At short times after startup, the transient microviscosity scales as $\eta^{\text {micro }}(t) / \eta \sim t^{1 / 2}$ as shown in the log-log plot given in Fig. 5-in agreement with the analytical solution (15). 
No matter what the Péclet number diffusion always dominates at short times ( $\Delta x_{\text {diffusion }} \sim \sqrt{\Delta t}$ vs $\Delta x_{\text {advection }} \sim \Delta t$ ). The curves for various $P e$ start to separate as the structure forms. The disturbance is communicated through the suspension by particle collisions-at higher $P e$, the probe moves farther in a given time and thus propagates the disturbance more quickly. Force-thinning behavior observed in steady-state suspensions is evident, as the long-time plateau decreases with increasing $P e$.

At long times, the microviscosity asymptotes to a steady value. These values are compared to the results for the steady-state microviscosity given by Squires and Brady (2005) in Table I. As seen in the table, the agreement between the long-time behavior obtained via our solution of Eq. (26), and that obtained via a steady-state analysis, is very good.

\section{B. Relaxation of the microstructure: Cessation behavior}

The decay in velocity after shutoff is plotted in Fig. 6 and, as was found in the analytical solution in Sec. IV, an initially rapid decay of the stress at very short times is followed by a slower long-time decay, indicating at least two relaxation modes. The rate of decay also varies with the initial condition: The higher the pre-cessation $P e$, the faster the relaxation. While it seems counterintuitive that a more strongly deformed microstructural disturbance would decay faster than a weakly deformed one, this behavior can be understood by recalling that in order to relax the stress, the probe need only diffuse the length of the boundary layer. That distance scales as $P e^{-1}$, so the higher the Péclet number, the faster the initial relaxation. Nearly all stress relaxation occurs during this process, owing to the dependence of the bath-particle drag on the contact value of the microstructure [cf. Eq. (6)]. At longer times, the Brownian diffusion of the bath particles continues and acts to close the wake and restore isotropy, which occurs on a time scale set by how long it takes a bath particle of size $b$ to diffuse laterally into the wake of width $a, \tau \sim a^{2} / D_{b}$, driven by the gradient in particle density across the wake. In this long-time limit, the majority of the microstructural relaxation occurs with very little change in probe speed; the stress relaxation is exponential, $\sim e^{-t}$, signaling the approach to equilibrium.

Were the probe moving alone through the solvent, the curves in Fig. 6 would drop instantaneously to zero upon shutoff of the external force. But in the presence of a microstructure (of passive particles), the probe continues to move after the external force is removed, as if a force still acted on it-or as if the probe has encoded information about its motion into the microstructure, and this information persists for some time.

The temporal persistence, or storage, of the dispersion stress can be viewed as the duration of the microstructural memory. In Fig. 7, the transient microviscosity has been multiplied by the Péclet number, and time re-scaled advectively. Comparison to the asymptotic result (25) shows agreement between analytical and numerical solutions at short advective times. At long times, the curves collapse for all $\mathrm{Pe}$ onto one curve-signaling the loss of memory of the initial condition. The duration of microstructural memory decreases with increasing $P e$, indicating that the information about the probe's motion is stored entropically in the distorted microstructure. The more dominant the Brownian motion, the longer the duration of the memory of the initial condition.

\section{BROWNIAN DYNAMICS SIMULATION}

Up to now, we have studied the transient behavior of colloidal particles theoretically by solving the pair Smoluchowski equation. To simplify our analysis, an assumption of diluteness was made. But an alternative approach is to examine the detailed motion of the individual particles by dynamic simulation; no assumption of diluteness is then 
required. Here, the dynamics are governed by the Langevin equation, a stochastic force balance that includes Brownian, external, hydrodynamic, and other interparticle forces:

$$
\mathbf{m} \cdot \frac{d \mathbf{U}}{d t}=\mathbf{F}^{H}+\mathbf{F}^{e x t}+\mathbf{F}^{B}+\mathbf{F}^{P},
$$

where $\mathbf{m}$ is the mass (or moment of inertia) tensor and $\mathbf{U}$ is the particle velocity. On the right-hand side are the forces that act on a particle, which include the hydrodynamic drag $\mathbf{F}^{H}$, along with external, Brownian, and interparticle forces, $\mathbf{F}^{e x t}, \mathbf{F}^{B}$, and $\mathbf{F}^{P}$, respectively. For the excluded annulus model (cf. Fig. 2), the hydrodynamic drag is given by $\mathbf{F}^{H}=-6 \pi \eta a \mathbf{U}$ and the external force $\mathbf{F}^{\text {ext }}=\mathbf{0}$ for all particles except the probe. The external force is prescribed, the Brownian force is given by

$$
\overline{\mathbf{F}^{B}}=0, \quad \overline{\mathbf{F}^{B}(0) \mathbf{F}^{B}(t)}=2 k T\left(6 \pi \eta a_{i}\right) \mathbf{I} \delta(t),
$$

and $\mathbf{F}^{P}$ is the hard-sphere interactive force between particles (cf. Sec. II A). The overbar denotes an average over times long compared to the solvent time scale, and the Brownian impacts are instantaneously correlated, with $\delta(t)$ the Dirac delta function. Particle sizes are given by $a_{i} \equiv a$ for the probe and $a_{i} \equiv b$ for a bath particle. Because the particles are small, inertia can be neglected and the left-hand side of Eq. (29) is zero. The velocity of the particle $\mathbf{U}=\left(\mathbf{F}^{\text {ext }}+\mathbf{F}^{B}+\mathbf{F}^{P}\right) / 6 \pi \eta a_{i}$ can then be integrated forward in time over a step $\Delta t$ to obtain a particle's displacement. In dimensionless form, this equation reads

$$
\Delta \mathbf{X}=-P e \hat{\mathbf{u}} \Delta t+\Delta \mathbf{X}^{B}+\Delta \mathbf{X}^{P}
$$

where $\hat{\mathbf{u}}$ is the unit vector antiparallel to the direction of the external force, $\hat{\mathbf{u}} \equiv-\mathbf{F}^{\text {ext }} / F^{\text {ext }}$. Time is made dimensionless on the diffusive time scale, $\tau_{D} \sim a^{2} / D_{b}$, and lengths are scaled with particle size, $\Delta X \sim a_{i}$. The Brownian displacement obeys the statistics

$$
\overline{\mathbf{X}^{B}}=0, \quad \overline{\mathbf{X}^{B}(0) \mathbf{X}^{B}(t)}=2 \Delta t \mathbf{I},
$$

giving a Brownian displacement at each time step that scales as $\Delta X \sim \sqrt{2 \Delta t}$. The dimensionless external force is given by $P e=F^{e x t} /(k T / b)$. When $P e \gtrsim 1$, time is rescaled advectively, $\tau_{a d v} \sim \tau_{D} P e^{-1}$, in order to resolve the larger displacements occurring over a given time step. To allow a direct comparison between simulation and theory, time is rescaled post-simulation to match the scaling set in the theory in Sec. II C: $\tau_{D} \sim(a+b)^{2} / D_{r}=(a+b)^{2} / D_{F}$. Similarly, displacements are rescaled to match the theory: $\Delta X \sim(a+b)$.

To begin the simulation, a probe of size $a$ is placed among a randomly distributed bath of particles of size $b$ in the simulation cell. At each time step, every particle is given a Brownian displacement of random direction, simulating a continuum Newtonian solvent of viscosity $\eta$ and density $\rho$ with thermal energy $k T$. The probe particle is displaced at each time step by a distance $P e$ in the direction of the external force; this force and its direction remain constant until the force is shut off. When two particles contact one another, the hard-sphere collision is treated via a "potential-free" algorithm [Heyes and Melrose (1993); Foss and Brady (2000); Carpen and Brady (2005)]. The overlap between two particles is prevented by displacing and separating the pair along their line of centers until they are no longer in contact. These hard-sphere collisional displacements $\Delta \mathbf{X}^{P}=-\Delta X^{P} \mathbf{n}$ are directed along the line of centers $\mathbf{n}$ of the colliding pair and weighted inversely 
according to the size of the colliding particle. For a complete description of Brownian dynamics of active microrheology, see Carpen and Brady (2005).

The stochastic nature of the Brownian force and hard-sphere collisions requires many realizations of the startup and cessation flow in order for the probe to sample a sufficient number of bath configurations. Thus, we measure the average displacement over each time step $\Delta t_{i}$ where $i=1,2, \ldots M$ and $M$ is the number of time steps in a simulation. The corresponding displacement over the $i$ th time step, $\Delta t_{i}$, is $\Delta \mathbf{X}_{i}\left(\Delta t_{i}\right)$ and is then averaged across many simulations

$$
\left\langle\Delta \mathbf{X}\left(\Delta t_{i}\right)\right\rangle=-P e\left\langle\hat{\mathbf{u}}\left(\Delta t_{i}\right)\right\rangle \Delta t+\left\langle\Delta \mathbf{X}^{P}\left(\Delta t_{i}\right)\right\rangle,
$$

where the Brownian displacement is zero on average across realizations, and the angle brackets $\langle\cdot\rangle$ signify an average across multiple simulations. The probe velocity over any given time step is the displacement divided by the length of the time step; the average total velocity and the hard-sphere velocity are given by $\left\langle\mathbf{U}_{i}\right\rangle=\left\langle\Delta \mathbf{X}_{i}\right\rangle / \Delta t_{i}$ and $\left\langle\mathbf{U}_{i}^{P}\right\rangle=\left\langle\Delta \mathbf{X}_{i}^{P}\right\rangle / \Delta t_{i}$, respectively:

$$
\left\langle\mathbf{U}\left(\Delta t_{i}\right)\right\rangle=-P e\left\langle\hat{\mathbf{u}}\left(\Delta t_{i}\right)\right\rangle+\left\langle\mathbf{U}^{P}\left(\Delta t_{i}\right)\right\rangle .
$$

Equation (34) shows that the mean speed $\langle U\rangle$ of the probe is the speed it would have if traveling alone in a solvent plus a reduction in its speed $\left\langle U^{P}\right\rangle$ due to the hindrance of the other particles. As before (cf. Sec. II A), we interpret the reduced mean speed as an effective viscosity of the dispersion. Recall the definition of the effective viscosity for $\mathrm{CF}$ given by Eq. (3). In that equation, we now have $\langle U\rangle=U^{0}+\left\langle U^{P}\right\rangle$ and $U^{0}=F^{e x t} / 6 \pi \eta a$, where the bath particles slow the probe's mean speed, $\left\langle U^{P}\right\rangle<0$. Thus in the CF case, the mean speed of the probe provides a measure of the effective viscosity

$$
\frac{\eta_{F}^{e f f}}{\eta}=\left[1+\frac{\left\langle U^{P}\right\rangle}{F^{e x t} / 6 \pi \eta a}\right]^{-1}
$$

and the reduction in mean speed gives a measure of the particle contribution to the viscosity, $\eta_{F}^{P}$,

$$
\frac{\eta_{F}^{P}}{\eta} \equiv-\frac{\left\langle U^{P}\right\rangle}{F^{e x t} / 6 \pi \eta a}, \quad \mathrm{CF} .
$$

The subscript $F$ indicates CF mode. Finally, in the case of a dilute bath in the CF case, the interparticle contribution to the viscosity measured in simulation is identical to the microviscosity defined by theory,

$$
\frac{\eta_{F}^{P}}{\eta}=\frac{\eta^{\text {micro }}}{\eta}, \quad \phi_{b} \ll 1
$$

In the $\mathrm{CV}$ case, the probe velocity $\mathbf{U}$ is held fixed while the probe's force fluctuates as it encounters another particle: $\langle\mathbf{F}\rangle=6 \pi \eta^{e f f} a \mathbf{U}^{e x t}$. The corresponding expression for the effective viscosity in the $\mathrm{CV}$ case may be written as

$$
\frac{\eta^{e f f}}{\eta}=\frac{\langle F\rangle}{6 \pi \eta a U^{e x t}} .
$$


Here, $\langle F\rangle=F^{0}+\left\langle F^{P}\right\rangle$ and $F^{0}=6 \pi \eta a U^{e x t}$. Thus, we have that $\eta^{\text {eff }} / \eta=1+\left\langle F^{P}\right\rangle /$ $6 \pi \eta a U^{e x t}$ and

$$
\frac{\eta_{U}^{P}}{\eta}=+\frac{\left\langle F^{P}\right\rangle}{6 \pi \eta a U^{e x t}}, \quad \mathrm{CV}
$$

The velocity $\left\langle\mathbf{U}^{P}\right\rangle$ and force $\left\langle\mathbf{F}^{P}\right\rangle$ are the quantities that are directly computed in simulation and theory, and measured in experiment: In the CF case, one measures the reduction in probe speed $\left\langle\mathbf{U}^{P}\right\rangle$ due to collisions with the background bath particles; and in the $\mathrm{CV}$ case, the additional force $\left\langle\mathbf{F}^{P}\right\rangle$ required to maintain constant probe velocity as it interacts with the another particle. Thus, the quantities $\eta_{F}^{P}$ and $\eta_{U}^{P}$ are the computationally and experimentally measured analogues of the microviscosity $\eta^{\text {micro }}$ defined in the (dilute) theory. In subsequent sections, the theoretical prediction of $\eta^{\text {micro }}$ is compared to the particle viscosity $\eta^{P}$ measured in simulation.

In the present study, an external force is suddenly applied to the probe starting at $t=0$ and is sustained for a period of time sufficient to allow the microstructure to evolve to steady state. The external force is then abruptly shut off, and the suspension allowed to relax as the Brownian motion of the particles continues. A range of forcing strengths was studied, with a focus on strong departures from equilibrium: $P e=10, P e=30$, and $P e=100$. The bath-particle volume fraction was varied from dilute to concentrated: $\phi_{b}=0.10, \phi_{b}=0.35$, and $\phi_{b}=0.45$. An ensemble of 18000 independent simulations of this startup/cessation sequence was performed for each parameter combination; the large number is needed because statistical data are obtained from the probe's motion only. The length-to-width size ratio of the (periodic) simulation cell was increased as the Péclet number increased, to assure that the probe would not re-enter its own wake. A longer cell allows the trailing wake behind the probe to fully recover an isotropic structure via Brownian motion of the bath particles before the probe exits one periodic cell and enters the next. The number of bath particles per simulation, at a given volume fraction $\phi_{b}$, was increased with cell length to allow the proper uniformity in the distribution of particles. This number ranged from 300 to 600 . To mirror the theoretical work described above, the probe- to bath-particle size ratio $a / b$ is set to unity. Both $\mathrm{CF}$ and $\mathrm{CV}$ modes were studied, and the time-dependent particle viscosity for the entire startup and cessation regimes was then computed via Eq. (36) for CF and Eq. (39) for CV and plotted in Fig. 8; the results are discussed below.

To gain insight into the relationship between transient viscosity and structure, the spatial distribution of bath particles around the probe was recorded at key points in time"density snapshots" taken from three orthogonal slices of the simulation cell, each of thickness $a$, passing through the probe center (Fig. 9). Each slice provides an instantaneous image of the microstructure as might be viewed via optical techniques in a physical experiment, one view each from the three orthogonal directions in a frame of reference moving with the probe. Two of the views are identical, due to the axisymmetry around the probe: The first (and second) is a side-view of the simulation cell transverse to probe forcing; the images shown are taken from this viewpoint. The third is a view looking along the direction of probe forcing (and is not presented). The density measurement was taken at eight selected times aimed toward understanding key features in the transient viscosity curves.

\section{A. Constant external force}

In Sec. IIC, two approaches in nonlinear microrheology were discussed: constantforce microrheology and constant-velocity microrheology. In this section, a constant 


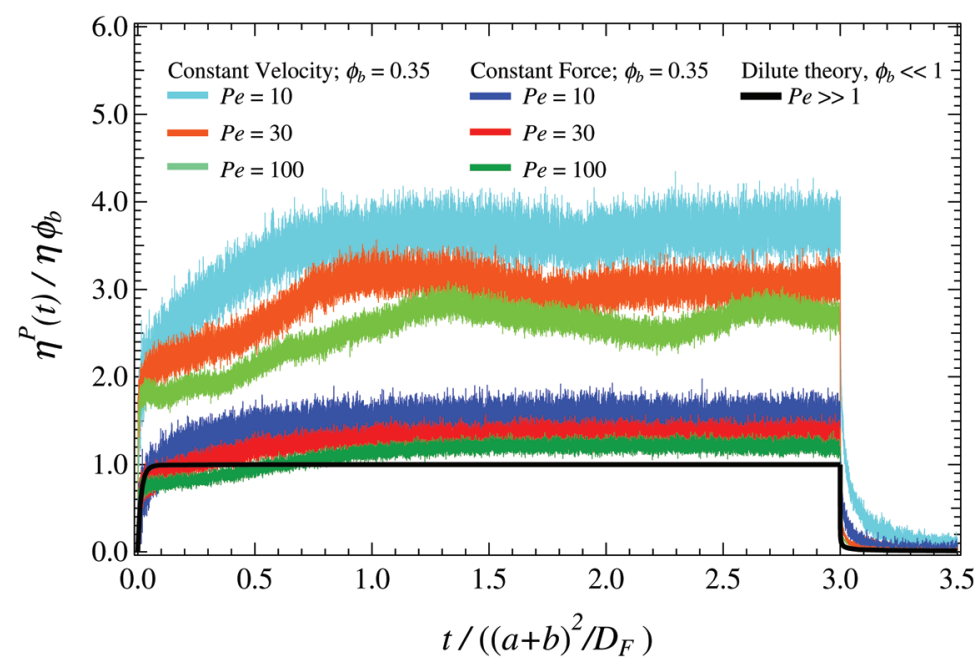

FIG. 8. Brownian dynamics: Transient microviscosity versus time for startup and cessation. Top three curves: $\mathrm{CV}$; bottom three curves: CF. $P e$ as shown; volume fraction of bath particles $\phi_{b}=0.35, a / b=1$. Forcing starts at $t=0$ and is shut off at $t=3$. Corresponding density snapshots for selected times are shown in Fig. 9. Details of post-cessation curves shown in Fig. 10.

external force is applied to the probe with sudden onset at $t=0$ at the beginning of the simulation. As the probe begins to move, it deforms the surrounding particle arrangement. The deformation of the microstructure evolves as the probe's motion continues, resulting in a change in the drag on the probe. Probe forcing is sustained until $t=3.0$ and then is instantaneously removed.

Equation (36) gives the particle viscosity from the measured hard-sphere velocity; it is compared to the dilute-theory prediction for $P e \gg 1$-the bottom three curves in Fig. 8

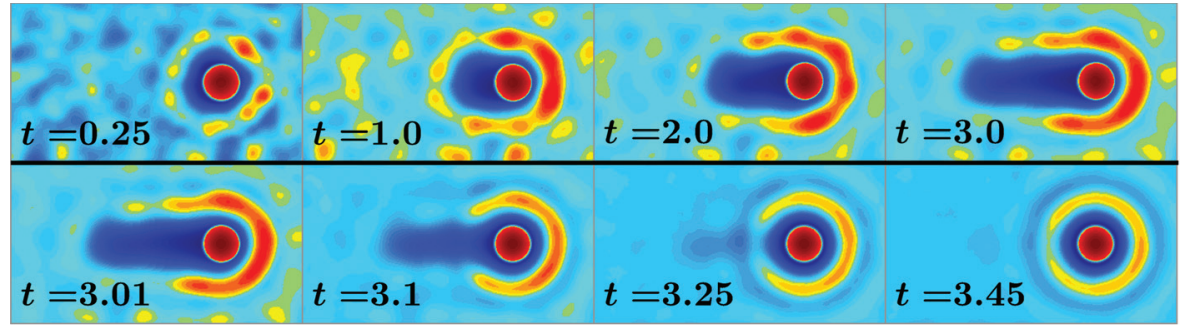

(a) Constant velocity (CV)

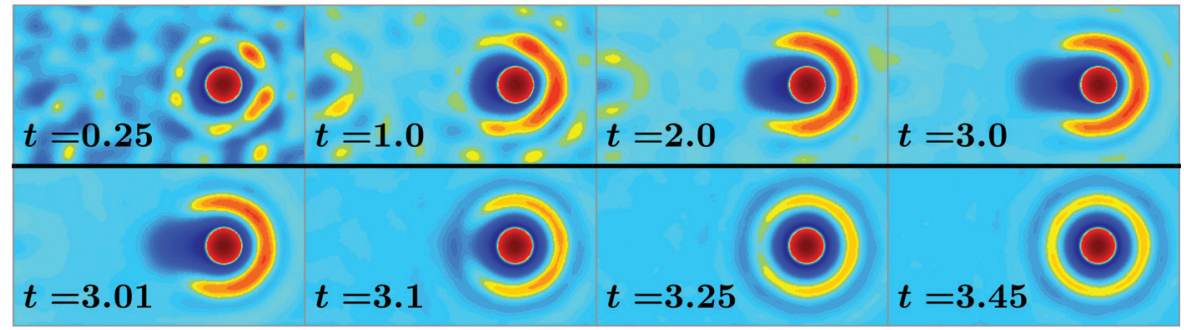

(b) Constant force (CF)

FIG. 9. Microstructural evolution for (a) CV, (b) CF, with startup (cessation) in first (second) row of each. $P e=30, \phi_{b}=0.35$. Times shown correspond to Fig. 8. [A closeup view of post-cessation viscosity curves $(t>3)$ is given in Fig. 10.] 
(dark blue, red, and dark green traces). In order to connect the evolving stress to changes in structure, density snapshots for $P e=30, \phi_{b}=0.35$ are shown in Fig. 9(b)-where the first four frames correspond to the startup of flow, and the second four frames correspond to relaxation after the force is shut off. The time at which each snapshot is taken is inset in each snapshot.

Before the flow is turned on, the microstructure is at equilibrium. Probe motion is started and the viscosity rises sharply, as seen in Fig. 8. During this rapid ascent, the equilibrium microstructure is disrupted; particles collide and propagate the force, and the boundary-layer shape characteristic of high- $P e$ flows begins to emerge $[t=0.25$ and $t=1.0$, respectively, in Fig. 9(b)]. The initial ascent scales as $t^{1 / 2}$ where the first step taken by the probe is diffusive. At intermediate times, $t \lesssim 1$, the boundary layer begins to form on the time scale of the flow, $(a+b) / U \sim \tau_{D} / P e$. This boundary-layer formation time $\tau_{B L}$ should decrease with the number of collisions, which scale with the volume fraction of bath particles; the scaling thus predicts that $\tau_{B L} \sim \tau_{D} / P e \phi_{b}$. In Fig. 8, the 1/Pedependence of the early climb to steady state is confirmed, as increasing values of $P e$ shift the transition from short- to long-time behavior left to earlier times.

At long times, $t \gtrsim 1.0$, the probe has moved its size and its mean speed has plateaued, signifying the approach to steady state. The force(shear)-thinning behavior observed in steady-state suspensions is also evident here, as the long-time plateau decreases with increasing $P e$.

The viscosity appears to reach approximately $90 \%$ of its asymptotic value within one diffusive time, and an asymptotic value by $t=3.0$, for both modes. However, the density plots in Fig. 9(b) indicate that the wake is still evolving at $t=3.0$. To confirm whether the viscosity had reached steady state at $t=3.0$, additional simulations for both $\mathrm{CV}$ and $\mathrm{CF}$ were performed for a startup duration of ten diffusive times. ${ }^{2}$ The majority of stress evolution again occurred within one diffusive time; this was followed by a much slower growth toward the (zero-slope) plateau viscosity by $t=8.0$ and $t=4.0$ for the $\mathrm{CV}$ and CF cases, respectively. The time for formation of the upstream boundary layer at contact coincides with attainment of $\sim 90 \%$ of the steady-state viscosity; while the downstream wake continues to evolve for a much longer time, it produces little change in the stress. The physical mechanisms underlying the multiple relaxation modes, and how they determine the approach to steady state, are explored further in Sec. VIC.

The dilute theory and the Brownian dynamics measurements for concentrated baths agree at high $P e$, as shown in Fig. 8. A comparison also shows that the long-time value of the viscosity depends on concentration: The dilute suspension is less viscous. But importantly, the time to reach steady state appears to be independent of volume fraction. We expect the theory prediction (18) to hold for all volume fractions (not too close to maximum packing.) Insensitivity to volume fraction of the time to steady state indicates that pair interactions dominate stress formation. Thus, the simple two-particle theory captures the physics relevant to stress formation and relaxation, regardless of concentration, as must be so, because the boundary layer sets the contact value, which is a two-particle interaction.

The external force is turned off at $t=3.0$ and relaxation continues via Brownian motion of the particles. The post-cessation viscosity is shown in Fig. 10, where multiple relaxation modes can be seen. First, a precipitous drop in the stress occurs immediately after shutoff at $t=3.0$; as seen in the figure, more than $90 \%$ of the stress relaxes over a

\footnotetext{
${ }^{2}$ Due to heavy computational expense, longer duration startup simulations were performed $P e=30$ only. The data are plotted in Fig. 15 given in the Appendix.
} 


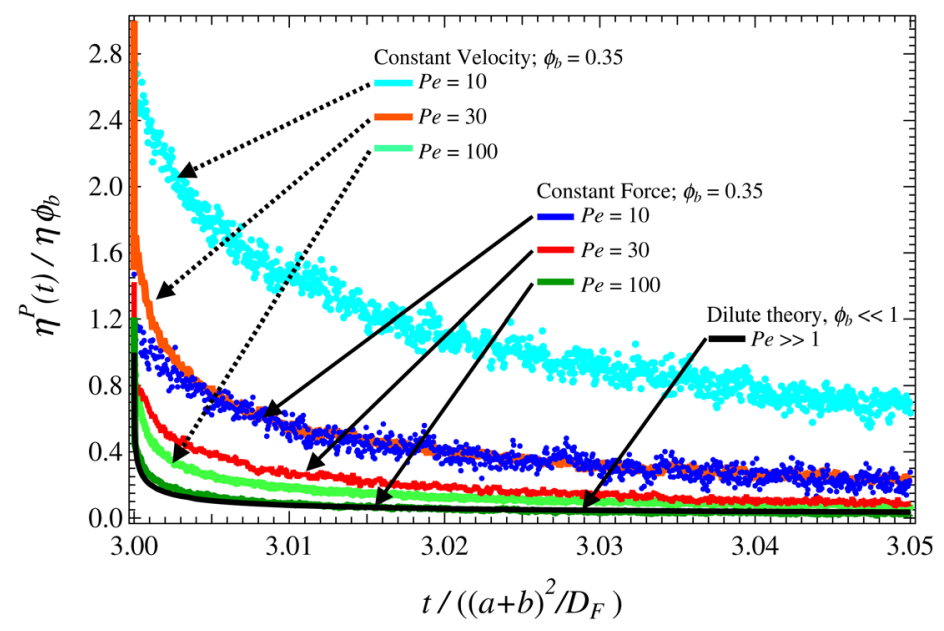

FIG. 10. Brownian dynamics: Transient microviscosity versus time for post-cessation. Force mode (CV and $\mathrm{CF}$ ) and $P e$ as shown; volume fraction of bath particles $\phi_{b}=0.35, a / b=1$. Forcing is shut off at $t=3$. Dottedline arrows point to CV curves; solid arrows point toward CF curves.

very short time, confirming the theory results reported in Sec. V. As the Brownian motion of the particles continues, the microstructure undergoes extensive rearrangement as it relaxes to equilibrium [density snapshots $t=3.1, t=3.25$, and $t=3.45$ in Fig. 9(b)], with very little change in the stress.

\section{B. Constant external velocity}

Instead of applying a constant external force to the probe, which allows its velocity to fluctuate, one may instead constrain the probe to move at a constant velocity through the bath. The difference in the two forcing modes has interesting dynamical consequences. In $\mathrm{CV}$ mode, the probe cannot adjust its speed but must press on through the bath-it cannot fluctuate to allow particles to clear out of its path and, in consequence, one expects the probe will build up a larger accumulation of particles on its upstream face and leave a more strongly depleted or longer wake. (In contrast, a CF probe will adjust its velocity if it encounters regions of higher or lower density.) The theory predicts that a higher boundary-layer density will lead to higher drag [cf. Eq. (6)]. In the dilute limit, this increase is expected to be a factor of 2 for a size ratio of unity (cf. Sec. II C).

The particle viscosity is determined from the hard-sphere velocity using Eq. (39). The results are given by the top three curves (cyan, orange, and light green traces) in Fig. 8 for a range of $P e$ and for a volume fraction of bath particles $\phi_{b}=0.35$. An important qualitative difference for the $\mathrm{CV}$ mode emerges in the startup regime. As seen in the figure, the microviscosity is nonmonotonic in time. The overshoot is evident for all $P e$ but becomes more pronounced as $P e$ increases. The microstructural origin of this behavior can be seen in the density snapshots in Fig. 9(a). The first snapshots $(t=0.25)$ for the CV and CF modes are indistinguishable. However, the next snapshots at $t=1.0$ show important differences: A longer wake and an unbroken ring of nearest neighbors downstream in the CV microstructure. This residual "cage" around the probe effectively increases its size and hence the drag. As the cage breaks open $(t=2.0)$, the drag in Fig. 8 settles to its steady value. While this behavior is clearly evident for CV forcing, it is not entirely absent in the CF case as seen by the slight overshoot in the viscosity for $P e=100$ (dark- 
green trace). In the corresponding CF density snapshots, a weak downstream residual structure appears to be shed-the downstream-portion of the ring.

At long times after startup, two key predictions of the theory are confirmed: First, the steady-state value of the CV viscosity is approximately double that for the CF case and second, the suspension force (shear) thins with increasing $P e$. The simulation results are in excellent agreement with our theoretical predictions (Secs. III B and V A) and with the steady-state, dilute-theory prediction by Squires and Brady that $\eta_{U}^{\text {micro }}=2 \eta_{F}^{\text {micro }}$. That the concentrated simulation results agree so well with our dilute theory gives support to the simple pair-level model. The data also compare well with the steady-state results of Carpen and Brady (2005), who conducted Brownian dynamics simulations at steady state and compared the steady values of $\eta_{F}^{P}$ and $\eta_{U}^{P}$. Interestingly, a slow, weak oscillation in the microviscosity can be seen clearly in the CV curves in Fig. 8. In CV mode, although the total probe velocity is fixed, the contribution to the velocity due to particle collisions necessarily fluctuates; this fluctuation is more pronounced as the probe encounters bath particles in the evolving transient structure. One can imagine a weak oscillation of the probe within the forming wake which damps out at longer times as the structure reaches steady state (as seen in Fig. 15 in the Appendix).

The microstructural features associated with the difference in viscosity can be seen in the density snapshots of Fig. 9(a). The particle accumulation on the upstream face of the probe is stronger and the trailing wake double in length compared to the corresponding CF-mode plot [Fig. 9(b)]. This is consistent with the notion of the CV probe as a "bulldozer" that pushes particles out of its way without slowing down. The consequence is a higher viscous resistance (and stronger fluctuations, as discussed below). Comparison of the long-time microstructures for CF versus CV $(2.0 \leq t \leq 3.0)$ shows that differences in microstructure persist at steady state, even when the balance between advection and diffusion is fully established. The constraint that the probe move at constant velocity leads to stronger upstream accumulation - and more pronounced anisotropy, viz., the increased length of the detached boundary layer. Because particle density must be conserved, a correspondingly longer depletion wake follows the probe.

The fluctuations of the particle viscosity are stronger in CV mode than in CF mode, as seen by the width of the traces in Fig. 8. Physically, the higher CV fluctuations result from the higher concentration of particles in its boundary layer which give rise to more frequent strong collisions. This difference also emerges naturally from the difference in diffusive scaling between the two modes. In fact, Squires and Brady (2005) estimated the velocity fluctuations for both cases, resulting in a predicted ratio for $\mathrm{CV}$ to $\mathrm{CF}$ of 2 . A coarse measurement of the width of the traces in Fig. 8 shows agreement with this prediction.

The flow is shut off at $t=3.0$, and as in the CF case, a very rapid drop in the stress is followed by a slower long-time decay (a closeup view of post-cessation viscosity curves is given in Fig. 10). The rate of stress relaxation depends on the pre-cessation value of $P e$ : The stress drops markedly faster for higher $P e$ than for lower $P e$. For higher $P e$, the boundary layer is thinner; the higher the Péclet number, the shorter the distance that must be traversed by the bath particles in order to relieve the stress. While the mechanism for relaxation, Brownian motion, is the same in the $\mathrm{CV}$ as in the $\mathrm{CF}$ case, for $\mathrm{CV}$ the probe's velocity is constrained to be identically zero at shutoff and only the bath particles diffuse. It is the relative diffusivity that sets the boundary-layer relaxation time scale. In the CV case, only the Brownian motion of the bath particles contributes to relaxation, and

$$
D_{U}=\frac{k T}{6 \pi \eta b}, \quad \mathrm{CV}
$$


and sets the time to relax the boundary layer. In the CF case, diffusivity is doubled (for $a=b)$,

$$
D_{F}=\frac{k T}{6 \pi \eta}\left(\frac{1}{a}+\frac{1}{b}\right), \quad \mathrm{CF},
$$

as the probe can also diffuse and relax the boundary layer. In consequence, stress relaxation is slower in the $\mathrm{CV}$ case. The freedom of the probe to fluctuate in the CF case not only reduces steady-state drag but also allows a faster stress relaxation and smaller fluctuations about the mean.

But the relaxation of the structure at long times is quite similar for the two cases. It can be seen in Fig. 9 that although the CV wake is twice the length of the CF wake, and the $\mathrm{CV}$ anisotropy is more pronounced, in both cases the equilibrium structure is nearly fully restored over one Brownian time-a much shorter time for relaxation than the evolution to steady state. This is discussed further in Sec. VIC.

Over long times, the wake closes and isotropy is restored. The relaxation mechanism that sets the time scale for wake closure is that of the bath particles diffusing laterally into the wake. The strong gradient in bath-particle density across the wake assures a high probability that the first Brownian step taken by particles nearby are in the direction of wake closure. This is seen clearly in the figure as the wake narrows in each progressive snapshot. The length of the wake does not change the lateral migration into it, but the more pronounced microstructural anisotropy in CV mode requires longer times for particles to distribute randomly around the probe.

It is natural to ask if the stress and structural evolution of the two modes can be made identical by a simple re-scaling of time; this is explored next by a detailed comparison of $\mathrm{CV}$ and $\mathrm{CF}$ simulation results.

\section{Structural evolution and relaxation time scales}

As noted above, the stress reaches $90 \%$ of its plateau value within one diffusive time for both CV and CF modes (cf. Fig. 8). The majority of the stress forms early in the evolution of the structure; while configurational changes continue for long times, the stress increases very little. This can be understood by recognizing that there are two primary relaxation modes: Diffusion within the boundary layer and diffusion in the wake. Boundary-layer formation time was discussed above; here we shall explore the subsequent differences in stress and structural evolution for CF versus CV modes, from the viewpoint of the relaxation time scales set by the respective diffusivities. The dilute theory predicts that differences in steady-state behavior arise due to the halving of the CV self-diffusivity. To understand what role this difference plays in the rate of approach to (and decay from) steady state, let us compare the $\mathrm{CV}$ microstructure at a given time $t$ to the CF microstructure at $2 t$ during startup and determine if a shift in time scales can make the CV and CF structural evolution quantitatively identical.

In Fig. 11, density snapshots are shown for both modes, side by side, for a startup duration of ten diffusive times, for $P e=30$ and $\phi_{b}=0.35$. In the second snapshot for $\mathrm{CV}$ mode, at $t=1.0$, the $\mathrm{CV}$ wake length roughly matches that for $\mathrm{CF}$ at $t=2.0$ - except for one distinct difference: The trailing nearest-neighbor ring in the CV density, which we associate with the viscosity-overshoot behavior. At longer times, the nearest-neighbor ring in the $\mathrm{CV}$ structure vanishes; although the wake lengths still differ, the structural shapes for $\mathrm{CV}$ at $t=2.0$ and $\mathrm{CF}$ at $t=4.0$ are similar. We expect also that the difference in structural relaxation time scale will lead to an earlier attainment of steady state for the $\mathrm{CF}$ bath. It can be seen that the CF microstructure reaches steady state by $4.0<t<6.0$, 


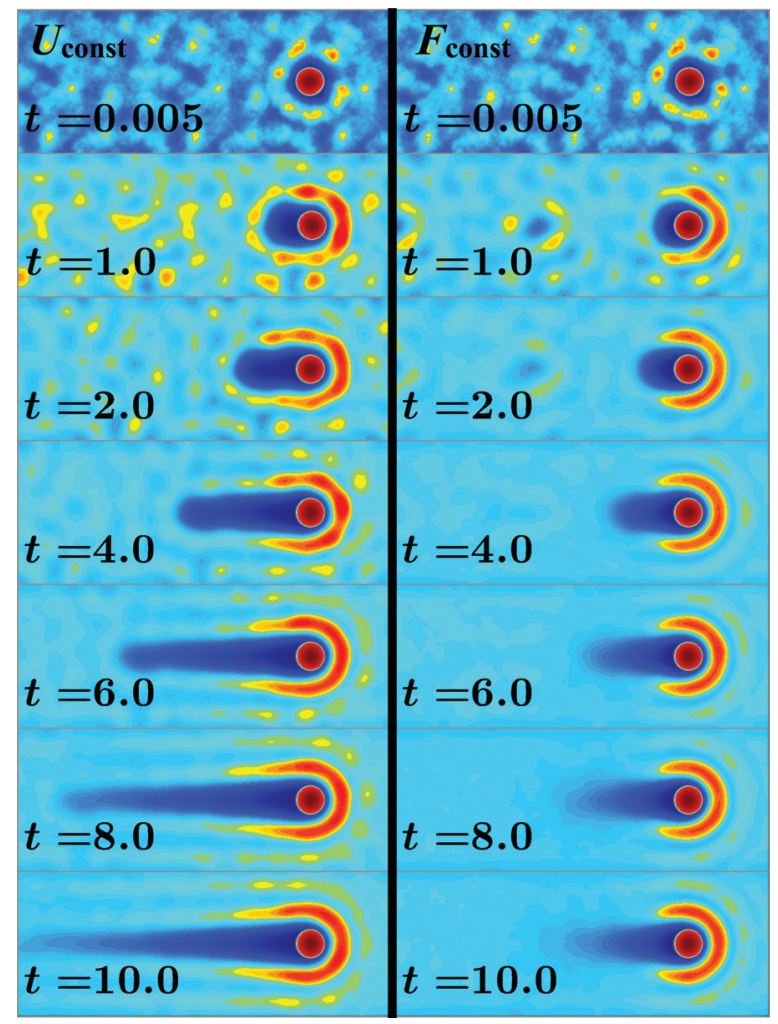

FIG. 11. Evolution of the microstructure during ten diffusive times for both forcing modes for $P e=30$ and $\phi_{b}=0.35$. CV is shown in left column; CF in right column. Dimensionless time is shown in each frame; both $\mathrm{CV}$ and $\mathrm{CF}$ time are scaled as in previous figures.

whereas it appears to just reach steady state for $\mathrm{CV}$ at $t=10.0$, indicating that the time to form the wake for $\mathrm{CV}$ is approximately double that for $\mathrm{CF}$.

The wake length is set by the imbalance between advection and diffusion: As the probe moves through the bath, it clears a path, leaving a depleted region behind it which is eventually healed by the Brownian motion of the bath particles. The length, $l_{w}$, after which the bath has fully regained isotropy is set by the speed $U$ at which the probe disturbs it and the time $\tau_{D}$ taken for bath particles to recover: $l_{w} \sim \tau_{D} U$. For fixed $P e$,

$$
l_{w} \sim P e D_{b} \tau_{D}
$$

for both modes. But if we prescribe equal numerical values of $P e$, the reduced diffusivity in $\mathrm{CV}$ gives a larger effective $P e$; thus

$$
l_{w}^{C V}=\left(1+\frac{b}{a}\right) l_{w}^{C F} .
$$

Visual inspection of the wake at $t=10.0$ confirms this estimate, where $a / b=1$. At steady state, the length of the $\mathrm{CV}$ wake is approximately double that for $\mathrm{CF}$.

The time to establish the wake is also set by the imbalance between advective and diffusive time scales. The probe can move its size in one advective time, $\tau_{a d v} \sim a / U \sim P e^{-1} a$; after one diffusive time, it can move $P e$ times its size. One then expects that the depletion 
wake behind the probe should be established in one diffusive time. But according to the density plots, it takes several diffusive time steps for the depletion region behind the probe to stop evolving and attain a steady configuration. Over the first diffusive time, the probe leaves a strongly depleted wake of length $a$. With each successive diffusive time, the probe creates another depleted region of size $a$ immediately behind it; a gradient in depletion pointing upstream shows that advection is won nearby the probe while diffusion is won by the bath particles downstream. In essence, to create the full wake, the depleted region must diffuse downstream. The time required scales as the wake length divided by the diffusive velocity of the bath particles,

$$
\tau_{w} \sim \frac{l_{w}}{\sqrt{D_{b} / \tau_{D}}}=\operatorname{Pe} \tau_{D}
$$

Although $O(P e)$ diffusive times are required to fully form the wake, relaxation and closure occurs within a single diffusive time. This is because during startup, the probe must encounter many other particles in order to achieve a steady balance between advection and diffusion. But during relaxation, many particles need to execute only a single diffusive step to relax. One then expects hysteresis in repeated on/off cycles of the force. The difference in $\mathrm{CV}$ versus CF relative diffusivity then gives the prediction that

$$
\tau_{w}^{C V}=2 \tau_{w}^{C F} .
$$

Overall, a rescaling of time gives approximately equal wake lengths and formation times for the two cases. The remaining difference between the two modes that cannot be scaled out is the overshoot behavior during startup—but this is a difference that vanishes at long times after startup.

For the stress relaxation behavior, recall from Eqs. (36) and (39) that the viscosity in the simulation is determined by dividing the particle velocity $\left\langle U^{P}\right\rangle$ for $\mathrm{CF}$ and the particle force $\left\langle F^{P}\right\rangle$ for $\mathrm{CV}$ by the Péclet number. Scaling the CV viscosity by a factor of 2 then

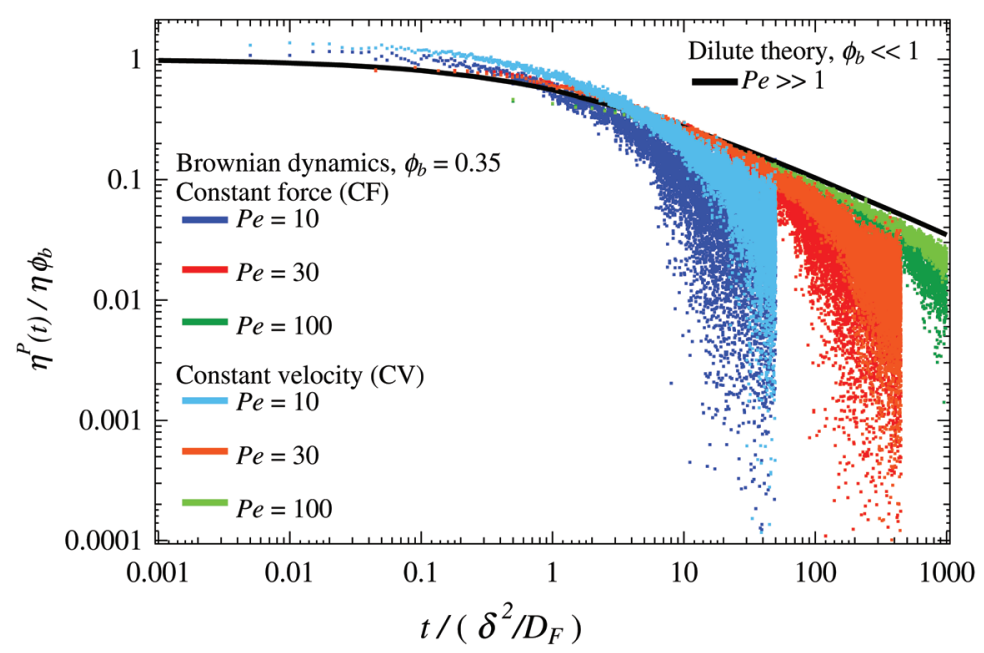

FIG. 12. Effect of concentration on relaxation behavior: Brownian dynamics simulation of concentrated bath (colored traces) compared to dilute theory (solid black curve). Both CF and CV viscosity plotted versus time made dimensionless on the CF diffusivity. CV traces divided by the diffusive scale-factor of 2 to illustrate decay from same initial value. 
gives the same effective Péclet number for the two cases, and it can be seen that the corresponding $\mathrm{CV}$ and $\mathrm{CF}$ traces for the values of $P e$ shown in Fig. 12 agree (the CF tracers become noisier at long times as the probe is allowed to fluctuate as well as the bath particles). The dilute theory relaxation prediction (24) is plotted alongside the concentrated simulation results in the same figure. The decay rates are identical-that is, the rate of relaxation of the microstructure and the stress are independent of the volume fraction. This notable result demonstrates that the simple two-particle theory is an accurate model for concentrated systems. From a practical point of view, it is useful, e.g., to set the cutoff time at which steady state measurements can be taken in experiments. From a more fundamental point of view, it reveals that structural evolution is a primarily pair phenomenon.

\section{CREEP RECOVERY BEHAVIOR}

Elastic behavior in suspensions arises due to the presence of a deformed particle microstructure. Particles accumulate (and deplete) in space because they hinder one another's movement along a flow line; when flow is stopped, the compressed structure relaxes over time if Brownian motion or interparticle forces are present. In the current study, the Brownian force is the driving force: Statistically, two particles that are pushed together and then released will be driven apart by Brownian motion - the gradient in their distribution gives rise to an entropic force that acts to make uniform their spatial distribution. Similarly, in a suspension of particles, applying a load to a set of particles deforms their distribution, restricting the number of available configuration states of the particles. The reduction in entropy exacts a free-energy penalty; this free-energy (density) is stored in the particle configuration and results in an increase in suspension stress. Removal of the external load allows the particles to regain a uniform distribution, which releases the stored free energy and reduces the stress. Some of this energy release is elastically recoverable as the suspension "springs" back, while some of the energy is dissipated viscously into the solvent. In contrast, a single particle in pure fluid dissipates all of its energy on

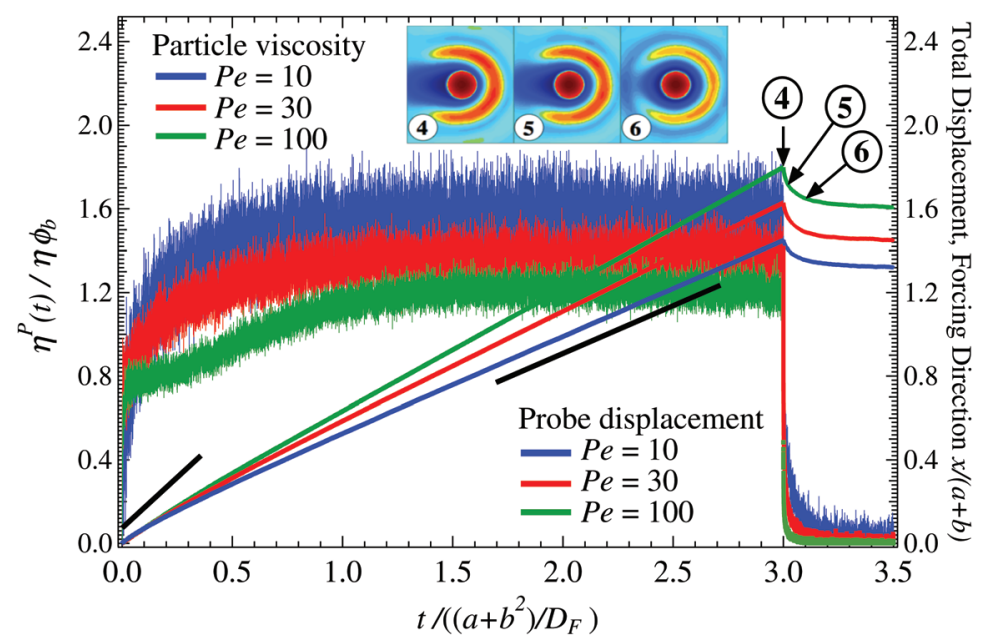

FIG. 13. Displacement of the probe during startup and cessation: Brownian dynamics simulation, constant external force. Displacement (colored solid lines) and viscosity (colored traces) as a function of time. Black line segments indicate change in displacement slope from intermediate to late times during startup. Data are for $P e$ as shown and volume fraction of bath particles $\phi_{b}=0.35$. Forcing starts at $t=0$ and is shut off at $t=3.0$. 
any time scale longer than its momentum relaxation time. Stress in a hard-sphere dispersion therefore gives some measure of its ability to temporarily store free energy.

To explore this behavior, the absolute position of the probe was monitored during simulation at CF. It is plotted in Fig. 13 alongside the constant force particle viscosity as a function of time for $P e=10, P e=30$, and $P e=100$ and $\phi_{b}=0.35$. During startup $(0 \leq t \leq 3.0)$, two distinct slopes can be seen (denoted by the short black line segments). The initially higher speed of the probe slows during the first diffusive step taken by the probe, as the microstructure begins to deform. The long-time slope is smaller and constant as the microstructure approaches steady-state. The reduction in the rate of probe displacement signals the increase in drag, also shown in the plot. This can be thought of as a time-dependent force-thickening of the suspension. Recall that when hydrodynamic interactions are present in a dispersion, lubrication interactions between the probe and bath particles produce doublets and clusters that lead to shear(force)-thickening [Bergenholtz et al. (2002)]. Their larger effective size reduces the average material velocity at the given imposed force, i.e., increases the viscosity. Here, in the absence of hydrodynamic interactions, the effective size of the probe changes from an initially isolated particle to a doublets or multiplets, as the structure deforms around it. Shear (force) thickening is primarily the result of particle cluster formation and these form temporarily even in the absence of hydrodynamic interactions.

Upon removal of the external force $(t=3.0)$, the probe travels backward, as seen in Fig. 13. The suspension "remembers" configurations from the past for a finite period of time; removal of the force allows the probe to return to a previous position, an important manifestation of nonlinear elasticity-memory-in colloidal dispersions.

We recall from Fig. 10 that the majority of the stress in the suspension relaxes very quickly upon shutoff; it was shown that the mechanism for this relaxation was the motion of the probe in the boundary layer. For $P e=30$, the back-travel should be approximately the boundary-layer thickness $\delta \sim(a+b) / P e \approx 0.033(a+b)$. A coarse measurement of the "slope" of the back-travel region in Fig. 13 indicates that the initial back-travel distance (from point 4 to point 5 in the figure) is approximately $0.03(a+b)$, at which point much of the stress has relaxed. As the microstructure continues to relax, the majority of the probe back travel, $\Delta x \approx 0.154(a+b)$, occurs by $t=0.15$ after shutoff (point 6 ). The corresponding density plots are inset in the figure. The stress relaxation, travel distance, boundary-layer thickness, and density plots agree. The initial back-travel shows that much of the stress relaxation is due to the separation of the probe from the boundary layer. The remaining back travel is driven by the diffusion of bath particles as they recover isotropic distribution and entropically entrain the probe during relaxation.

The probe experiences this entropic "spring" force as a real force, which can be computed as $\mathbf{F}=\mathbf{R} \cdot \mathbf{U}$, where the back-travel velocity $\mathbf{U}$ can be estimated graphically from measurements of probe displacement in the figure as $U \approx \Delta x / \Delta t$. At short times after shutoff,

$$
F \approx \frac{k T}{b} \frac{\Delta x}{\Delta t} \approx 5.41 \frac{k T}{b}
$$

giving the back-travel of the probe that occurs by the time most stress relaxation has taken place (point 6). The entropic force can also be estimated theoretically as $F \sim k T \nabla \log g$. At infinite dilution, in the limit of strong forcing, this becomes

$$
F \approx \frac{k T}{b}
$$


for a size ratio of unity, where we have used the value of $g(1, P e \rightarrow \infty)$ from dilute theory [Squires and Brady (2005)]. The simulation results for the concentrated bath (46) and the dilute theory prediction above thus agree qualitatively, as they must. The dilute theory prediction (47) was also compared to simulation results with $P e=30$ but with volume fraction of bath particles $\phi_{b}=0.1$; here $F \approx 1.49 \mathrm{kT} / \mathrm{b}$. The restoring force acting on the probe in the more concentrated bath is higher as the osmotic force scales with the number of particles nearby.

One can also view the restoring force as a measure of the osmotic compressibility of the bath, $F /(k T / b) \sim \Pi / n k T$. The Carnahan-Starling equation of state expresses the osmotic compressibility $Z=\Pi / n k T$ in terms of the volume fraction of particles as

$$
Z=\frac{\Pi}{n k T}=\frac{1+\phi_{b}+\phi_{b}^{2}-\phi_{b}^{3}}{\left(1-\phi_{b}\right)^{3}},
$$

which predicts $Z\left(\phi_{b}=0.10\right)=1.52$ and $Z\left(\phi_{b}=0.35\right)=5.21$, in close agreement with the entropic spring force estimated above. Here, the probe is a passive particle being carried along during the relaxation of the dispersion and provides a direct measure of osmotic compressibility. (Note that since the probe and bath particles have the same size $(a=b)$, once the force is removed, the probe becomes indistinguishable from a bath particle.)

\section{COMPARISON TO MACRORHEOLOGY}

We have suggested that the force/velocity relationship in microrheology is analogous to the stress/strain-rate relationship in macrorheology and that both approaches should yield the same qualitative information about structural evolution and relaxation. In order to test this assertion, we compare here the findings of the present study with the findings of previous macrorheology studies.

Transient suspension behavior was studied by dynamic simulation by Foss (1999), where Brownian and Stokesian dynamics were used to study the startup and cessation behavior of suspensions undergoing shear flow. At early times after the onset of shear

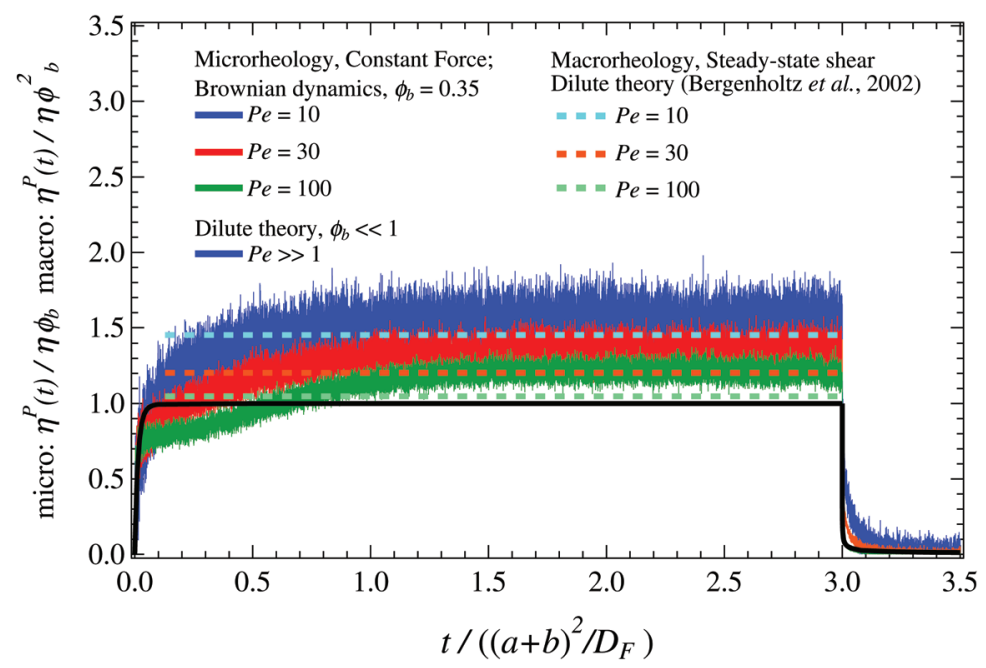

FIG. 14. Comparison of long-time (steady-state) pair-contribution to the particle viscosity for microrheology via Brownian dynamics in concentrated suspensions and via dilute theory, versus macrorheology via the dilute theory of Bergenholtz et al. (2002). 
flow, Foss also finds that the viscosity grows as $t^{1 / 2}$ (in the absence of hydrodynamic interactions). He also observed similar overshoot behavior in the startup regime, which increases in amplitude as $P e$ increased. At long times, he reported the same shearthinning behavior as steady state was reached. Each of these three fundamental behaviors was recovered here via nonlinear microrheology.

After shutoff, both micro- and macrorheology reveal qualitatively identical behavior: Relaxation occurs over at least two time scales. The first is the steep relaxation in stress due to diffusion in the boundary layer, which occurs in a time set by the boundary-layer thickness $t \sim O\left(\delta^{2} / D\right)$. Both studies also find that this is followed by a longer-range relaxation of the structure, which takes a time of order $\sim\left(a^{2} / D\right)$ required for particles to travel their size.

To compare micro- to macrorheology at steady state, results for a sheared, dilute suspension as obtained by theory [Bergenholtz et al. (2002)] are shown alongside the results of the present study in Fig. 14, where the pair-level particle contribution to the steadystate shear viscosity is shown by the dashed lines. The steady-state values obtained via microrheology in a concentrated bath are slightly higher than the corresponding dilute results; results for the dilute microrheology theory is also shown and are in excellent agreement with the macrorheology theory.

\section{CONCLUSIONS}

The formation and relaxation of nonequilibrium stress in colloidal dispersions was studied using the framework of nonlinear microrheology. In this approach, a dilute dispersion of hard-sphere colloids is driven far from equilibrium by the motion of an externally forced Brownian probe particle. In order to explore time-dependent behavior of the structure and stress, the velocity and displacement of the probe were monitored during startup of the flow for several diffusive time steps. Forcing was then abruptly removed, and monitoring of probe displacement and velocity were continued after shutoff as the suspension relaxed.

During the startup of the flow, the microstructure evolves initially as a one-dimensional diffusive monopole in time, and the viscosity grows as $t^{1 / 2}$. Importantly, the early-time behavior of the transient microviscosity is the same regardless of the strength of probe forcing. This is because the first collision results from a diffusive step that scales as $\sim \sqrt{\Delta t}$, which at short times is larger than an advective step that scales as $\sim \Delta t$. Thus, the first change in the viscosity propagates diffusively, in keeping with the connection between the short-time self-diffusivity and the infinite-frequency (zero shear-rate) viscosity. As the probe encounters more particles, it slows down and the drag on the probe asymptotes to a long-time steady value. The force-thinning characteristic of hard-sphere suspensions at steady state was recovered at long times. These values also agreed with the steady-state values reported by Squires and Brady (2005), Khair and Brady (2006), and Carpen and Brady (2005). Analytical and numerical results and Brownian dynamics simulation measurements were in good agreement-a somewhat surprising result, given that the simulation baths were far from dilute. This indicates that pair interactions dominate the development of both structure and stress and that the simple dilute theory proposed gives a strong model even for concentrated suspensions.

After the forcing is removed, the motion of the probe and suspension do not cease instantaneously. The suspension relaxation occurs over at least two time scales: First, an initially rapid decay in stress that accompanies diffusive relaxation of the boundary layer. The rate of initial decay $\tau_{B L} \sim \delta^{2} / D$, where $\delta \sim P e^{-1}$, and thus is set by the pre-cessation Péclet number. Stronger departures from equilibrium recover faster. While this behavior is found for both constant-force and constant-velocity forcing modes and the underlying 
mechanism is the same for the two cases, the time scales differ quantitatively. For the former, both the probe and the bath particles can diffuse and relax through the boundary layer to relieve stress; whereas in the latter case, only the bath particles can diffuse, halving the initial relaxation rate for equally sized probe and bath particles and resulting in a slower stress relaxation. In either case, the stress relaxation time depends on the boundary-layer thickness, which it must, because the primary contribution to hard-sphere suspension stress is that due to direct particle interactions. Importantly, the dilute theory captures the physics of the relaxation process: The pair-level equation obtained for the relaxation of the viscosity was in agreement with the concentrated suspension in the simulations (cf. Fig. 12) the relaxation rate of the boundary layer is evidently independent of volume fraction. These results are consistent with similar findings by Foss (1999). In contrast to the stress, the majority of the structural relaxation occurs on a longer time scale, where wake closure occurs by gradient-driven diffusion of bath particles laterally across the wake.

A comparison was made between $\mathrm{CF}$ and $\mathrm{CV}$ regimes. Brownian dynamics simulations confirm the steady-state prediction by Squires and Brady that $\eta_{U}^{\text {micro }} \sim 2 \eta_{F}^{\text {micro }}$. Brownian dynamics simulation of steady-state microrheology by Carpen and Brady (2005) found similar values for the viscosity as the long-time startup results in this study. Physically, in CV mode, the probe velocity (and path) cannot fluctuate, which results in a larger accumulation of bath particles in the boundary layer and thus a stronger resistance to probe motion. The time to reach steady state in $\mathrm{CV}$ mode is also longer because the time required to sample all configurations of the microstructure is longer, due to the need to encounter many different spikes and dips in density, which is reflected in the halving of the diffusive time scale. An overshoot in the stress in the CV case becomes more pronounced as $P e$ increases. The underlying mechanism for this behavior was found by comparing images of the evolving microstructural density. The cage of nearest-neighbor bath particles around the probe remains intact in the downstream region for several diffusive time steps. This residual cage around the probe effectively increases its size and hence the drag. As the cage breaks open, the drag settles to its steady value. This overshoot behavior is closely connected to the cluster formation that is the underlying mechanism for shear-thickening in suspensions [Bergenholtz et al. (2002)].

Additional differences between the two modes include a longer wake in the CV mode, a denser boundary layer, and a halving of the initial stress relaxation rate after shutoff. But it was also shown that re-scaling of time and length with the appropriate relative diffusivity removes these differences.

Creep recovery after shutoff provides insight into the connection between stress, freeenergy storage, and entropic memory. During startup and at steady state, the probe's motion compresses the particle distribution, restricting its entropy; the free-energy change required to do so is released by the microstructure as it relaxes and regains access to more configuration states. As seen when the force is abruptly removed, not all of the strain can be recovered; in the case of $P e=30$, the entropic spring force was approximately $5 k T / b$ or one-sixth the applied force. The remaining energy is dissipated viscously to the solvent by the probe and the bath particles. The storage is entropic in origin (not enthalpic), and this is the origin of nonequilibrium suspension stress.

While the amount of energy density stored in the structure increases with increasing $P e$, the duration of this storage decreases with increasing $P e$. The two phenomena have a common origin: Memory decay occurs via Brownian motion; at high $P e$, Brownian motion is too weak to dissipate probe energy, and storage is high; but upon shutoff, only a small region must relax and the stored energy is dissipated quickly. But when $P e$ is small, Brownian motion easily dissipates the energy of steady probe motion, and storage is low; yet upon shutoff, a comparatively large region (more particles) must relax. A more 
revealing way to view this connection between magnitude and duration of stress is to examine the ratio of recovered to input energy. For $P e=10, P e=30$, and $P e=100$, this ratio is approximately $30 \%, 18 \%$, and $7 \%$, respectively for a volume fraction of $\phi_{b}=0.35$. Thus, as $P e$ increases, a decreasing fraction of input energy can be stored in the distorted particle configuration, and the duration for which this storage persists is shorter.

The connection between the entropic force and the osmotic compressibility supports this view of the stress as energy storage. An interesting question that arises here is whether the entropic spring force [cf. Eq. (46)] can be derived from first principles; at steady state, the statically distorted microstructure generates this entropic force suggesting that $F^{\text {entropic }} \sim k T \nabla \log g$, where $g$ is the nonequilibrium pair-distribution function.

The comparison between transient nonlinear macrorheology and microrheology shows that in both cases, the early stress evolution scales with the square root of time and shows the same shear (force) thinning over a range of $P e$ [Foss (1999)]. In both micro- and macrorheology, the long-time relaxation behavior collapses onto a single curve, indicating that memory of the initial condition is lost.

Several questions remain. The effect of hydrodynamic interactions was not studied in this investigation. When hydrodynamic interactions are important, shear thickening occurs for large $P e$ at steady state. Because hydrodynamic interactions introduce an additional dissipative mechanism, the early scaling of the microviscosity will change. Lubrication forces drive the relative mobility between a pair of particles to zero which leads to a plateau at low frequency - the high shear/flow-rate viscosity. Upon shutoff of the force, the hydrodynamic contribution to the stress will decay instantaneously.

The mechanisms that underlie the constant-force versus constant-velocity probe regimes should be studied with a view toward the force fluctuations that arise during CV motion. While the velocity-velocity autocorrelation gives insight into diffusivity, what can we learn from force-force correlation-for example, about the viscosity? The forcevelocity cross-correlation, $\langle\mathbf{U F}\rangle$, can be viewed as a measure of work done on the system; since this total energy must be either stored or dissipated, it would make sense to view $\langle\mathbf{F F}\rangle$ and $\langle\mathbf{U U}\rangle$ in terms of (nonequilibrium) fluctuation and dissipation. And while the size ratio $a / b$ was taken to be unity for this study, it would be interesting to explore the parameter space of variable size ratio and understand its contribution to relaxation time scales. A qualitative change is not expected, except perhaps when hydrodynamic interactions are important.

Finally, we turn to the idea that probe motion is a window through which we can view the connection between fluctuation and dissipation. What can nonequilibrium fluctuation and dissipation tell us more fundamentally about energy and motion? Stress relaxation away from equilibrium depends strongly on the probe's ability to fluctuate. Although power input by the probe must all be dissipated, it takes time to do so. Energy is stored temporarily as increased suspension stress. In our recent work [Zia and Brady (2012)], it was shown that the stress is given by the balance between nonequilibrium fluctuations and viscous relaxation in nonlinear microrheology. The strength of advective forces relative to diffusive forces - the Péclet number-is equivalently a ratio of diffusive to advective time scales. It sets the time scale over which the microstructural disturbance persists; $P e$ is thus a memory parameter in nonlinear flows - it indicates how long the microstructure "remembers" the disturbance caused by the probe's motion.

\section{ACKNOWLEDGMENTS}

This work was supported in part by the National Defense Science and Engineering Graduate Fellowship program and by the National Science Foundation grant number 


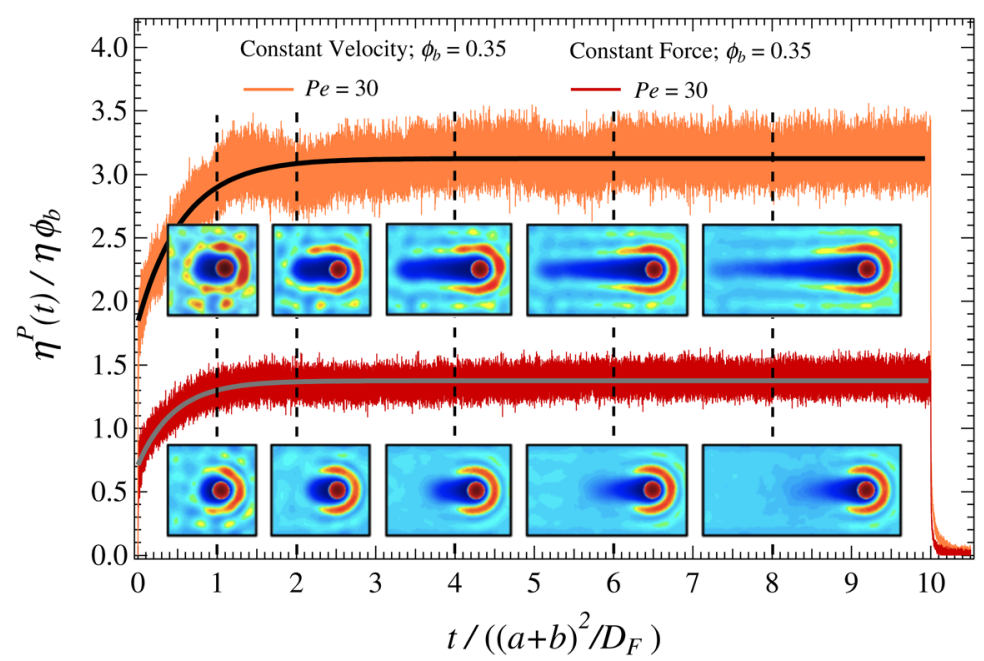

FIG. 15. Extended startup duration: Brownian dynamics simulation. Transient microviscosity versus time for startup and cessation. Top curve: CV; bottom curve: CF. $P e=30$; volume fraction of bath particles $\phi_{b}=0.35$, $a / b=1$. Forcing starts at $t=0$ and is shut off at $t=10$. Dashed lines connect the inset density snapshots to corresponding point in viscosity curve.

CBET-0931418. The authors thank Dr. James W. Swan for the Brownian dynamics code for microrheology.

\section{APPENDIX: LONG-TIME BEHAVIOR DURING STARTUP}

To confirm whether the viscosity had reached steady state at $t=3.0$, additional simulations for both $\mathrm{CV}$ and $\mathrm{CF}$ were performed for a startup duration of ten diffusive times. Due to heavy computational expense, longer duration startup simulations for only $P e=30$ were performed. The data are plotted in Fig. 15. The majority of stress evolution occurs within one diffusive time; this is followed by a much slower growth toward the (zero-slope) plateau viscosity by $t=8.0$ and $t=4.0$ for the CV and CF cases, respectively. As can be seen in the figure, the viscosity has reached steady state at the time of shutoff, $t=10$.

\section{References}

Abramowitz, M., and I. A. Stegun, Handbook of Mathematical Functions with Formulas, Graphs, and Mathematical Tables (Dover, New York, 1964).

Bausch, A. R., F. Ziemann, A. A. Boulbitch, K. Jacobson, and E. Sackmann, "Local measurements of viscoelastic parameters of adherent cell surfaces by magnetic bead rheometry,” Biophys. J. 75, 2038-2049 (1998).

Bergenholtz, J., J. F. Brady, and M. Vicic, "The non-Newtonian rheology of dilute colloidal suspensions," J. Fluid Mech. 456, 239-275 (2002).

Brady, J. F., "The rheological behavior of concentrated colloidal dispersions," J. Chem. Phys. 99, 567-581 (1993).

Brady, J. F., "The long-time self-diffusivity in concentrated colloidal dispersions,” J. Fluid. Mech. 272, 109133 (1994).

Brady, J. F., and J. F. Morris, "Microstructure of strongly sheared suspensions and its impact on rheology and diffusion,” J. Fluid. Mech. 348, 103-139 (1997).

Breedveld, V., and D. J. Pine, "Microrheology as a tool for high-throughput screening," J. Mater. Sci. 38, 44614470 (2003). 
Carpen, I. C., and J. F. Brady, "Microrheology of colloidal dispersions by Brownian dynamics simulations," J. Rheol. 49, 1483-1502 (2005).

Crocker, J. C., "Measurement of the hydrodynamic corrections to the Brownian motion of two colloidal spheres,” J. Chem. Phys. 106, 2837-2840 (1997).

Crocker, J. C., J. A. Matteo, A. D. Dinsmore, and A. G. Yodh, "Entropic attraction and repulsion in binary colloids probed with a line optical tweezer," Phys. Rev. Lett. 82, 4352-4355 (1999).

Crocker, J. C., and D. G. Grier, "Methods of digital video microscopy for colloidal studies," J. Colloid Interface Sci. 179, 298-310 (1996).

Crocker, J. C., M. T. Valentine, E. R. Weeks, T. Gisler, P. D. Kaplan, A. G. Yodh, and D. A. Weitz, “Two-point microrheology of inhomogeneous soft materials," Phys. Rev. Lett. 85(4), 888-891 (2000).

Einstein, A., "On the theory of the Brownian movement," Ann. Phys. 19, 371-381 (1906).

Foss, D. R., "Rheological behavior of colloidal suspensions: The effects of hydrodynamic interactions," Ph.D. thesis, California Institute of Technology, 1999.

Foss, D. R., and J. F. Brady, "Brownian dynamics simulation of hard-sphere colloidal dispersions," J. Rheol. 44(3), 629-651 (2000).

Freundlich, H., and W. Seifriz, "Über die elästizitaet von solen und gelen," Z. Phys. Chem. 104, 233-261 (1923).

Gisler, T., and D. A. Weitz, "Scaling of the microrheology of semidilute f-actin solutions," Phys. Rev. Lett. 82, 1606-1610 (1999).

Green, M. S., "Markov random processes and the statistical mechanics of time-dependent phenomena II," J. Chem. Phys. 22, 398-413 (1954).

Guilford, W. H., R. C. Lantz, and R. W. Gore, "Locomotive forces produced by single leukocytes in vivo and in vitro,” Am. J. Physio-Cell. Ph. 268(5), C1308-C1312 (1995).

Habdas, P., D. Schaar, A. C. Levitt, and E. R. Weeks, "Forced motion of a probe particle near the colloidal glass transition," Europhys. Lett. 67, 477-483 (2004).

Heyes, D. M., and J. R. Melrose, "Brownian dynamics simulations of model hard-sphere suspensions," J. NonNewtonian Fluid Mech. 46, 1-28 (1993).

Hocquart, R., and E. J. Hinch, “The long-time tail of the angular-velocity auto-correlation function for a rigid Brownian particle of arbitrary centrally symmetric shape," J. Fluid Mech. 137, 217-220 (1983).

Kaffashi, B., V. T. O'Brien, M. E. Mackay, and S. M. Underwood, "Elastic-like and viscous-like components of the shear viscosity for nearly hard sphere, Brownian suspensions," J. Colloid Interface Sci. 187, 22-28 (1997).

Khair, A. S., and J. F. Brady, “'icroviscoelasticity' of colloidal dispersions,” J. Rheol. 49, 1449-1481 (2005).

Khair, A. S., and J. F. Brady, "Single particle motion in colloidal dispersions: A simple model for active and nonlinear microrheology,” J. Fluid Mech. 557, 73-117 (2006).

Khair, A. S., and J. F. Brady, "Microrheology of colloidal dispersions: Shape matters," J. Rheol. 52, 165-196 (2008).

Kubo, R., “The fluctuation-dissipation theorem,” Rep. Prog. Phys. 29, 255-285 (1966).

Lau, A. W. C., B. D. Hoffman, A. Davies, J. C. Crocker, and T. C. Lubensky, "Microrheology, stress fluctuations, and active behavior of living cells," Phys. Rev. Lett. 91, 198101 (2003).

Levine, A. J., and T. C. Lubensky, "One- and two-particle microrheology,” Phys. Rev. Lett. 85, 1774-1778 (2000).

Lionberger, R. A., and W. B. Russel, "High frequency modulus of hard sphere colloids," J. Rheol. 38(6), 18851908 (1994).

Lu, Q., and M. J. Solomon, "Probe size effects on the microrheology of associating polymer solutions," Phys. Rev. E 66, 061504 (2002).

Mackay, M. E., and B. Kaffashi, "Stress jumps of charged colloidal suspensions, measurement of the elasticlike and viscous-like stress components," J. Colloid Interface Sci. 174, 117-123 (1995).

MacKintosh, F. C., and C. F. Schmidt, "Microrheology," Curr. Opin. Colloid Interface Sci. 4, 300-307 (1999).

Mason, T. G., and D. A. Weitz, "Optical measurements of frequency-dependent linear viscoelastic moduli of complex fluids,” Phys. Rev. Lett. 74, 1250-1253 (1995).

Mason, T. G., K. Ganesan, J. H. van Zanten, D. Wirtz, and S. C. Kuo, "Particle tracking microrheology of complex fluids," Phys. Rev. Lett. 79(17), 3282-3286 (1997). 
Meyer, A., A. Marshall, B. G. Bush, and E. M. Furst, "Laser tweezer microrheology of a colloidal suspension," J. Rheol. 50, 77-92 (2006).

Morris, J. F., and J. F. Brady, "Self diffusion in sheared suspensions," J. Fluid. Mech. 312, 223-252 (1996).

Nägele, G., and J. Bergenholtz, "Linear viscoelasticity of colloidal mixtures," J. Chem. Phys. 108, 9893 (1998).

Perrin, J -.B., "Mouvement Brownien, réalité moléculaire (Brownian motion and the molecular reality)," Ann. Chim. Phys. 18, 5-114 (1909).

Russel, W. B., "Brownian motion of small particles suspended in liquids," Annu. Rev. Fluid Mech. 13, 425-455 (1981).

Schultz, K. M., and E. M. Furst, "High-throughput rheology in a microfluidic device,” Lab Chip 11, 3802-3809 (2011).

Squires, T. M., and J. F. Brady, “A simple paradigm for active and nonlinear microrheology,” Phys. Fluids 17, 073101-1-073101-2 (2005).

Valko, P. P., and J. Abate, "Comparison of sequence accelerators for the Gaver method of numerical Laplace transform inversion,” Comput. Math. Appl. 5307, 1-10 (2002).

Watanabe, H., M.-L. Yao, K. Osaki, T. Shikata, H. Niwa, and Y. Morishima, "Nonlinear rheological behavior of a concentrated spherical silica suspension," Rheo. Acta 35(5), 443-445 (1996b).

Watanabe, H., T. Sato, and K. Osaki, "Relaxation of spherical micellar systems of styrene-isoprene diblock copolymers. 2. Nonlinear stress relaxation behavior," Macromolecules 29, 3890-3897 (1996a).

Wilson, L. G., A. W. Harrison, A. W. Schofield, J. Arlt, and W. C. K. Poon, "Passive and active microrheology of hard-sphere colloids," J. Phys. Chem. 113, 3806-3812 (2009).

Zia, R. N., and J. F. Brady, "Single particle motion in colloids: Force-induced diffusion," J. Fluid Mech. 658, 188-210 (2010).

Zia, R. N., and J. F. Brady, "Microviscosity, microdiffusivity, and normal stresses in colloidal dispersions," J. Rheol. 56(5), 1175-1208 (2012).

Ziemann, F., J. Radler, and E. Sackmann, "Local measurements of viscoelastic moduli of entangled actin networks using an oscillating magnetic bead micro-rheometer," Biophys. J. 66, 2210-2216 (1994). 